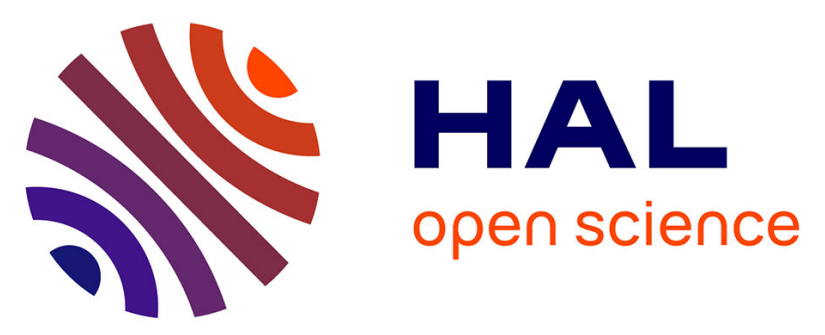

\title{
A radiolytic origin of organic matter in primitive chondrites and trans-neptunian objects? New clues from ion irradiation experiments
}

\author{
Mathilde Faure, Eric Quirico, Alexandre Faure, Philippe Boduch, Hermann \\ Rothard, Emmanuel Balanzat, Donia Baklouti, Rosario Brunetto, Lydie \\ Bonal, Pierre Beck, et al.
}

\section{To cite this version:}

Mathilde Faure, Eric Quirico, Alexandre Faure, Philippe Boduch, Hermann Rothard, et al.. A radiolytic origin of organic matter in primitive chondrites and trans-neptunian objects? New clues from ion irradiation experiments. Icarus, 2021, 364, pp.114462. 10.1016/j.icarus.2021.114462 . hal-03309043

\section{HAL Id: hal-03309043 https://hal.science/hal-03309043}

Submitted on 30 Jul 2021

HAL is a multi-disciplinary open access archive for the deposit and dissemination of scientific research documents, whether they are published or not. The documents may come from teaching and research institutions in France or abroad, or from public or private research centers.
L'archive ouverte pluridisciplinaire HAL, est destinée au dépôt et à la diffusion de documents scientifiques de niveau recherche, publiés ou non, émanant des établissements d'enseignement et de recherche français ou étrangers, des laboratoires publics ou privés. 


\section{A RADIOLYTIC ORIGIN OF ORGANIC MATTER IN PRIMITIVE CHONDRITES AND TRANS- NEPTUNIAN OBJECTS? NEW CLUES FROM ION IRRADIATION EXPERIMENTS}

Mathilde Faure ${ }^{1}$, Eric Quirico ${ }^{1 *}$, Alexandre Faure ${ }^{1}$, Philippe Boduch ${ }^{2}$, Hermann Rothard ${ }^{2}$, Emmanuel Balanzat ${ }^{2}$, Donia Baklouti ${ }^{3}$, Rosario Brunetto ${ }^{3}$, Lydie Bonal ${ }^{1}$, Pierre Beck ${ }^{1}$ and Bernard Schmitt ${ }^{1}$

${ }^{1}$ Université Grenoble Alpes, CNRS, Institut de Planétologie et Astrophysique de Grenoble (IPAG), UMR 5274, Grenoble F-38041, France

${ }^{2}$ Centre de Recherche sur les Ions, les Matériaux et la Photonique, CIMAP-CIRIL-GANIL, Normandie Université, ENSICAEN, UNICAEN, CEA, CNRS, 14000 Caen, France

3'Institut d'Astrophysique Spatiale (IAS), Université Paris-Sud, UMR 8617-CNRS INSU, Bât 121, F-91405 Orsay, France

*Corresponding author:

Eric Quirico

eric.quirico@univ-grenoble-alpes.fr

\section{Reference:}

Faure, Mathilde, Eric Quirico, Alexandre Faure, Philippe Boduch, Hermann Rothard, Emmanuel Balanzat, Donia Baklouti, Rosario Brunetto, Lydie Bonal, Pierre Beck and Bernard Schmitt (2020). A radiolytic origin of chondritic organic matter? an experimental test by ion irradiation experiments. Icarus, 364, 114462 [doi: 10.1016/j.icarus.2021.114462][ 


\begin{abstract}
We question here the radiolytic origin of (i) polyaromatic insoluble organic matter (IOM) recovered from primitive chondrites, and (ii) organics at the surface of reddish TransNeptunian Objects (TNOs), some minor planets and icy satellites. Organic synthesis by ion irradiation was investigated through experiments on a variety of targets: Polyethylene glycol 1450, lignin, cellulose and sucrose, exposed to low (C $40 \mathrm{keV}$ and Ne $170 \mathrm{keV}$ ) and high energy (C $12 \mathrm{MeV}, \mathrm{Ni} 17 \mathrm{MeV},{ }^{78} \mathrm{Kr} 59 \mathrm{MeV}$ ) ions. These experiments show that all carbonaceous precursors evolves towards a sp²-rich amorphous carbon (a-C) above a critical nuclear dose of $10_{-7}^{+10} \mathrm{eV}_{\text {atom }}{ }^{-1}$. A thorough review of the literature shows that this value applies for a large range of carbonaceous materials, including C-rich simple ices. Below this critical dose, irradiated targets are carbonized and transformed into cross-linked polymeric disordered solids, with abundant radiolytic olefinic and acetylenic bonds, but devoid of aromatic or polyaromatic species. Ion irradiation of simple compounds, e.g. ices, is thereby not a viable process to synthesize IOM. However, in the case of aromatic-rich precursors, swift heavy ions irradiation leads to polyaromatic materials, by bridging existing aromatic or polyaromatic units. In the context of Early Solar System, i.e. Galactic Cosmic Rays (GCR) irradiation during 10-20 Myr, the formation of chondritic IOM from simple ices mixed with interstellar Polycyclic Aromatic Hydrocarbons (PAHs) appears as a plausible mechanism. This scenario, based on the recycling of existing carbonaceous interstellar grains under lowtemperature conditions, would account for the heterogeneity of the $\mathrm{D},{ }^{15} \mathrm{~N}$ and ${ }^{13} \mathrm{C}$ isotopic fractionations at the molecular scale, and the preservation of deuterium hot spots that are highly sensitive to high-temperature conditions $\left(>300^{\circ} \mathrm{C}\right)$. At the surface of TNOs, $\mathrm{sp}^{2}$-rich amorphous carbons are formed by the implantation of GCRs and Solar wind ions. The electronic dose is also very high for an irradiation time of several Gyr ( $>100$ eV.atom $^{-1}$ ), leading to the formation of reddish disordered solids, provided that the surface contains a minimum abundance of carbonaceous species. Finally, $\mathrm{sp}^{2}$-rich amorphous carbons produced in the laboratory (e.g. the ACAR compound from Zubko et al., 1996) are fair analogues of the darkening agent produced by radiolysis.
\end{abstract}




\section{INTRODUCTION}

Primitive carbonaceous chondrites contain $\sim 3-5 \mathrm{wt} \%$ of organic matter (OM). This abiotic material is of primary importance as it sheds light on carbon chemistry that was operating either in the protosolar disk (PSD) or in the local interstellar medium before the birth of the Solar System. As a major carrier of $\mathrm{H}, \mathrm{C}, \mathrm{N}$ and $\mathrm{O}$ elements, it also provides insights into the volatile species that have been accreted by the Early Earth, through asteroidal collisions. Chondritic organic matter is usually divided into two types: Soluble Organic Matter (SOM) and Insoluble Organic Matter (IOM), whether they are soluble or insoluble in usual solvents, respectively (Sephton, 2002). SOM is a complex mixture of molecules classified into 25 classes, including carboxylic and amino acids, alkanes and small Polycyclic Aromatic Hydrocarbons (PAHs). The analysis of C1-C10 molecules points to the presence of all possible isomers, a decreasing abundance against the number of carbon atoms and $\delta \mathrm{D}, \delta^{15} \mathrm{~N}, \delta^{13} \mathrm{C}$ fractionations that are the proof of their non-terrestrial origin (Burton et al., 2012; Elsila et al., 2012; Sephton, 2002). High Resolution Mass Spectrometry analysis of Murchison (CM2) SOM extracts reveals a very complex mixture made of $\sim 10^{6}$ different molecules, with masses extending up to $800 \mathrm{u}$ and a global composition dominated by $\mathrm{H}, \mathrm{C}$ and $\mathrm{O}$, and minor $\mathrm{N}$ and $\mathrm{S}$ elements (Schmitt-Kopplin et al., 2010). IOM has received a growing interest at the beginning of the 2000s, and knowledge on its structure and composition has much progressed (see the review of Alexander et al., 2017). In short, its elemental composition shares similarities with type III terrestrial kerogens and its molecular structure is relatively disordered and polyaromatic. However, the polyaromatic units (1-4 rings) are highly substituted and connected through ramified aliphatic linkages made of short alkyl chains (Cody and Alexander, 2005; Cody et al., 2002; Gardinier et al., 2000; Remusat et al., 2005a, 2005b; Wang et al., 2005). It also bears bulk $\delta \mathrm{D}, \delta^{15} \mathrm{~N}$ and $\delta^{13} \mathrm{C}$ fractionations distinct from those displayed by terrestrial organic matter, and shows in particular local tiny regions ( $\sim 1 \mu \mathrm{m}$ across) named hot spot that display very high deuteration levels (Alexander et al., 2007, 1998; Busemann et al., 2006). IOM also contains radicals and radicaloids, whose spatial distribution and temperature dependence constitute a valuable tracer of their extraterrestrial origin (Binet et al., 2002, 2004; Delpoux et al., 2011; Gourier et al., 2008).

In the 80 s and 90s, the high D/H enrichments measured in IOM was mainly interpreted by synthesis through low-temperature ion-molecule reactions in Interstellar Medium (ISM) (Kerridge, 1999; Robert and Epstein, 1982). The discovery of abundant crystalline silicates in comets by ground-based and satellite infrared observations and later, by the Stardust mission, have challenged this view. Indeed, silicates are known to be predominantly amorphous in ISM (Wooden et al., 2000; Zolensky et al., 2006). Meanwhile, the so-called $3.4 \mu \mathrm{m}$ aliphatic band observed towards the galactic center, which had been assigned to IOM (Ehrenfreund et al., 1991), has been reassigned to hydrogenated amorphous carbons (Dartois et al., 2007). Alternate scenarios have then emerged, suggesting OM synthesis in the PSD (Remusat et al., 2006) or in the asteroidal parent body (Cody et al., 2011). Experimental simulations based on OM synthesis in cold plasma reactors or thermal degradation in hydrothermal cells have successfully fit some of the characteristics of IOM - but also failed in reproducing others (Cody et al., 2011; Kuga et al., 2015). To date, no scenario or experiment fulfills all existing constraints in terms of structure, chemical and isotopic composition.

Interestingly, these recent studies require high temperature conditions, which promote aromatization and the formation of a polyaromatic structure. This process is that operating in Earth sedimentary basins, generating coals and kerogens. In addition, the $\mathrm{sp}^{2}$ structure of chondritic IOM is also consistent with a thermal process (Quirico et al., 2014). However, high temperature conditions are inconsistent with the presence of deuterium hot spots, and for circumventing this issue multi-stage scenarios have been proposed, based on a first formation stage followed by transport and finally hydrogen/deuterium exchanges in a colder region of the protosolar disk (Rémusat et al, 2006; Kuga et al., 2015). These scenarios face several problems, such as the low abundance of $\mathrm{H}_{3}{ }^{+}$isotopologues in the cold mid-plane, the high temperatures in the PDR and molecular layers of the PSD, the temperature heterogeneity in the inner region of the protosolar disk or the poorly efficient chemical 
interactions between nebular gas and the interior of dust grains (Cleeves et al., 2014; Visser et al., 2018).

Nevertheless, in addition to heating processes, ion irradiation may play a role. In the local ISM and the outer region of the protosolar disk, gas and dust were irradiated by Galactic Cosmic Rays (GCRs) that triggered solid-state chemistry. Under such conditions, ion irradiation of ices and carbonaceous dust leads to complex disordered organic materials which, depending on the nature of precursor and irradiation conditions, might have a polyaromatic structure (Auger et al., 2015; Balanzat et al., 1995; Brunetto et al., 2009). Here, we investigate the effect of precursor composition and energy range of the ions through experiments conducted at GANIL (Grand Accélérateur National d'Ions Lourds, Caen France) and CSNSM (Centre de Sciences Nucléaires et de Sciences de la Matière, Orsay, France). The paper is organized as follows. We present in section 2 the precursors that were irradiated, the preparation protocols and experimental conditions. In section 3, we present the results obtained for two main irradiation conditions, dominated by (i) electronic and (ii) nuclear interactions of the impinging ions with the target. In section 4, these results are extrapolated to astrophysical conditions and the plausibility of $\mathrm{OM}$ formation is discussed. Our main conclusion is that synthesis through the radiolysis of ISM precursors by Galactic Cosmic Rays (GCR) is plausible and may be consistent with the main compositional and structural constraints of $\mathrm{OM}$. We also address the issue of organics evolution at the surface of TransNeptunian Objects through the effect of solar energetic particles and GCRs, and show that radiolysis is significant and may indeed account for reddening and darkening.

\section{MATERIALS AND METHODS}

\subsection{Samples}

Irradiation experiments were conducted on the following organic solids: PEG1450, sucrose, lignin and $\alpha$-cellulose. Sucrose was purchased from Sigma-Aldrish and purity was 99.5 $\%$ (HPLC grade). PEG 1450 was purchased from Fischer. There was no indication about purity, but expected impurities are precursor monomers and the polymeric initiator that disappear when the film is formed. $\alpha$-Cellulose was purchased from Sigma Aldrich (reference C8002, lot 050M0140V). It was a white powder, but no indication about purity was provided. Lignin-alkali was purchased from Sigma Aldrich (reference 370959, lot MKBF624V). It contains $64.1 \%$ of carbon, $1.4 \%$ of sulfur, and $\mathrm{pH}$ was 7.

PEG 1450 stands for polyethylene glycol with an average molecular mass of 1450 $\mathrm{g} / \mathrm{mol}$. It is a homopolymer formed of linear chains of about thirty $\mathrm{O}-\mathrm{CH}_{2}-\mathrm{CH}_{2}-$ monomers, terminated by $-\mathrm{OH}$ groups and parallel to each other. PEG 1450 is solid at room temperature, soluble in water and alcohols. Sucrose $\left(\mathrm{C}_{12} \mathrm{H}_{22} \mathrm{O}_{11}\right)$ is a molecule formed by the assemblage of glucose and fructose through a glycosidic bond. It is a crystalline monoclinic solid at room temperature and soluble in water. Lignin and cellulose are both insoluble natural polymers. Cellulose is composed of long linear chains of glucose units and is not soluble in usual organic solvents and water. It can be considered as a refractory material, i.e. solid at room temperature even under low-pressure conditions $\left(<10^{-5} \mathrm{mbar}\right)$. Lignin has a polyphenolic structure, i.e. it contains aromatic rings with $\mathrm{OH}, \mathrm{COOH}$ and ether groups, and most importantly, it is a cross-linked polymer. This 3D structure leads to total insolubility and a refractory qualification. Both lignin and cellulose should be considered as more complex and disordered materials than sucrose and PEG 1450, with very different macromolecular structures.

A key objective of this study is to investigate the formation of chondritic insoluble organic matter from the radiolysis of solid carbonaceous precursors. However, these latter are unidentified, and a key issue concern is the so-called precursor effect. In the case of artificial and natural thermal carbonization of organic solids, the initial composition, structure (e.g. maturity) or mixing state (e.g. dilution in the host rock) of a precursor control to various extent the nature of the carbonized sample (Bernard et al., 2010; Beyssac et al., 2004; Quirico et al., 2009). To investigate this effect in the case of radiolytic carbonization, we have selected the above series of four compounds with different structures and complexities. In addition, 
these precursors have been selected for the following reasons. First, they mimic putative oxygen-rich carbonaceous precursors and show varying degrees of volatility (PEG > sucrose $>$ cellulose > lignin), composition and structure. PEG is here a fair analogue of mixtures made of chondritic SOM, which however has a higher $0 / C$ ratio (0.5 versus 0.2$)$ and a much broader molecular diversity. To a lesser extent, its simple molecular structure makes it useful to get insights into the radiolysis of ices or simple organics. Second, sucrose is the precursor of saccharose coke used for long as a standard in carbon sciences, which resembles primitive chondritic IOM (Schmidt and Hinrichs, 2020). This compound is thus an interesting target for comparing thermal and radiolytic carbonization. Cellulose is chemically close to sucrose but more refractory. Last, lignin contains a significant fraction of mono-aromatic species and is therefore an interesting precursor of the polyaromatic chondritic IOM.

Sucrose, cellulose and lignin were prepared as pellets $(\varnothing 1 \mathrm{~cm} ; \sim 1-3 \mathrm{~mm}$ thick) by pressing ground material under vacuum $\left(10^{-2}\right.$ mbar $)$ with a hydraulic press, up to 250 bar for several hours. Thin films of PEG1450 were prepared with a POLOS SPIN150i spin-coater. PEG1450 was dissolved in ethanol (0.11 g.L-1), and few drops were deposited on a ZnSe window, which was maintained under a $900 \mathrm{rpm}$ rotation during 25 minutes. A 1-5 $\mu$ m thick film was obtained.

\subsection{Irradiation experiments}

Irradiation experiments are presented in Table 1 as two main groups. The first group includes low energy irradiations $(<500 \mathrm{keV})$ conducted at Centre de Sciences Nucléaires et de Sciences de la Matière (CSNSM, Orsay - France) with the ion implantation platforms SIDONIE and IRMA, using $40 \mathrm{keV}$ Carbon and $170 \mathrm{keV}$ Neon ions. They were run at room temperature under ultra-vacuum with no in situ monitoring. Under these conditions, ions and target mostly experience elastic interactions and ions are eventually implanted. Samples investigated were a PEG 1450 thin film, as well as thick sucrose and cellulose pellets. These samples were characterized ex situ by Raman spectroscopy. The second group of experiments includes irradiations performed at higher energy ( $>500 \mathrm{keV})$ at Grand Accélérateur National d'Ions Lourds (GANIL, Caen - France). Samples were irradiated under vacuum $\left(\mathrm{P}=10^{-6} \mathrm{mbar}\right)$ at room temperature. The first experiment was performed on PEG 1450 thin films with a 12 $\mathrm{MeV}$ Carbon ions beam, a flux of $\sim 5.10^{13}$ ions. $\mathrm{cm}^{-2} . \mathrm{s}^{-1}$ and a maximal fluence of $4.10^{14}$ ions.cm2. The thickness of the films was much smaller than the implantation length, and projectile ion-target interactions were mainly electronic. During a second experimental session, thick pellets of sucrose, lignin and cellulose were irradiated with a $17 \mathrm{MeV} \mathrm{Ni}$ ions beam and a maximum fluence of $7.10^{13}$ ions. $\mathrm{cm}^{-2}$. Here, Nickel ions were implanted in the samples and the evolution of the samples was triggered by electronic interactions along most of the track, followed by nuclear interactions during implantation at the very end of the track. All these samples were ex situ analyzed by Raman micro-spectroscopy. Two irradiation experiments were run on PEG 1450 thin films at very low temperature, $10 \mathrm{~K}$, with ${ }^{78} \mathrm{Kr}^{15+}$ at $0.76 \mathrm{MeV} / \mathrm{u}$ to investigate the effect of temperature. We finally note that the diameter of the core track of a swift ion is typically $10 \mathrm{~nm}$ across, which means that tracks overlap are expected above a fluence of $5 \times 10^{13}$ ions. $\mathrm{cm}^{-2}$. As a result, experiments may be not fully relevant to GCR irradiation conditions in which 2-ion event are unlikely.

\subsection{Sample analysis}

At GANIL, IR spectra of PEG1450 were collected in situ during irradiation with a Nicolet FTIR spectrometer (Magna 550), operating in the spectral range 5000-600 $\mathrm{cm}^{-1}$ with a $1 \mathrm{~cm}^{-1}$ spectral resolution. Ex situ Raman and infrared spectroscopy were performed at Laboratoire de Géologie de Lyon (ENS, Lyon - France) and IPAG (Université Grenoble Alpes, France), respectively. $532 \mathrm{~nm}$ Raman spectra were collected with a Horiba LabRAM HR800 instrument equipped with a Spectra Physic argon ion laser and a x100 objective. $244 \mathrm{~nm}$ Raman spectra were collected with a LabRAM HR800 operating with a frequency-doubled $\operatorname{Ar}^{+}(244 \mathrm{~nm})$ excitation and a $\mathrm{x} 40$ objective. The power at the sample was adjusted through time- 
resolved measurements and visual check (for extensive details see Quirico et al. (2020). Raman spectroscopy was applied to all samples produced in this study. It presents decisive advantages: (i) it is a direct probe of the $\mathrm{sp}^{2}$ structure that controls optical properties in the visible range; (ii) it is a powerful tool for characterizing disordered and amorphous carbons and (iii) it allows for direct comparison with cosmomaterials (Quirico et al., 2014, 2020) and previous irradiation experiments reported in literature (e.g. Costantini et al., 2002a; Ferini et al., 2004).

\section{RESULTS}

\subsection{Irradiation of PEG1450 with $12 \mathrm{MeV}$ Carbon ions}

Thin films of PEG 1450 were irradiated up to a maximum fluence of $410^{4}$ ions. $\mathrm{cm}^{-2}$. Samples were recovered at $1.5 \times 10^{13}, 6 \times 10^{13}, 1.5 \times 10^{14}, 2.4 \times 10^{14}$ and $4 \times 10^{14}$ ions.cm $^{-2}$ for ex situ analyses (Table 1). Naked eye observation shows the progressive coloring of the initially transparent film, from colorless, to yellow, to dark brown, pointing to the increase of conjugated $\pi$ bonds in the sample (Fig. A1). The solubility of the film (in methanol) also decreases progressively with increasing the irradiation dose.

The chemical composition and structure of the film was monitored by in situ IR spectroscopy (Fig. 1; Tab. 2). We basically observe a significant evolution of the initial spectrum with a dramatic decrease of the integrated band intensities, pointing to a significant sputtering effect. The initial spectrum displays a congested structure around $2900 \mathrm{~cm}^{-1} \mathrm{due}$ to the symmetric and asymmetric stretching of $\mathrm{CH}_{2}\left(v_{\text {as }} \mathrm{CH}_{2}\right.$ at $2950 \mathrm{~cm}^{-1}, v_{\mathrm{S}} \mathrm{CH}_{2}$ at $2885 \mathrm{~cm}^{-1}$ and $2890 \mathrm{~cm}^{-1}$ ) and to combinations between vibration modes of adjacent monomers in the helicoidal chain at 2700, 2740, 2805, 2824 and $2862 \mathrm{~cm}^{-1}$ (Miyazawa et al., 1962). Upon increasing the irradiation dose, this band changes due to the rise of the methyl groups signature and to disordering, until the stabilization of the ratio of the numbers of methylene and methyl groups $\mathrm{n}_{\mathrm{CH} 2} / \mathrm{n}_{\mathrm{CH} 3}$. In the so-called fingerprint regions $\left(1800-500 \mathrm{~cm}^{-1}\right)$, we observe the narrow bands due to the bending and deformations modes of $-\mathrm{CH}_{2}$, and to $\mathrm{C}-\mathrm{C}$ and $\mathrm{C}-\mathrm{O}-\mathrm{C}$ skeletal modes, which broaden, shift and finally merge into a broad bump centered at $\sim 1250$ $\mathrm{cm}^{-1}$ (Fig. 1). We also observe the appearance of new features at $\sim 1700 \mathrm{~cm}^{-1}(\mathrm{C}=0), \sim 1600$ $\mathrm{cm}^{-1}(\mathrm{C}=\mathrm{C})$ and $2110 \mathrm{~cm}^{-1}(\mathrm{C} \equiv \mathrm{C}$, possible contribution of $\mathrm{C}=\mathrm{C}=0)$. The $\mathrm{C}=\mathrm{C}$ and $\mathrm{C} \equiv \mathrm{C}$ bands continuously increase and reveal the progressive unsaturation of the material, in agreement with the (qualitative) color evolution. The abundances of $\mathrm{C}=\mathrm{O}$ and $\mathrm{OH}$ groups first increase and then decrease with increasing further the irradiation dose. The chemical evolution of the sample can be semi-quantitatively estimated by calculating the integrated absorbance of the $\mathrm{OH}$ band (3050-3600 $\mathrm{cm}^{-1}$ ), the aliphatic band due to the stretching modes of $\mathrm{CH}_{2}$ and $\mathrm{CH}_{3}$ groups $\left(2600-3000 \mathrm{~cm}^{-1}\right)$, the acetylenic $\mathrm{C} \equiv \mathrm{C}\left(2073-2133 \mathrm{~cm}^{-1}\right)$, the carbonyl $\mathrm{C}=0$ and olefinic $\mathrm{C}=\mathrm{C}$ bands (gaussian fit) and the skeleton modes $\left(1000-1200 \mathrm{~cm}^{-1}\right)$. Four stages can be identified from these data (Fig. 2, 3):

- Stage 1: no significant chemical evolution.

- Stage 2: the abundance of aliphatic groups decreases while that of unsaturated species increases. The material looses hydrogen and free radicals on adjacent carbon atoms, which recombine into unsaturated bonds. The broadening of the skeleton modes around 1000-1200 $\mathrm{cm}^{-1}$ reveals an increasing heterogeneity and disorder. The presence of $\mathrm{CH}_{3}$ and $\mathrm{C}=0$ groups point to chain scissions. Cross-linking happens between the polymeric chains.

- Stage 3: the $\mathrm{OH}$ group decreases until disappearance, and the acetylenic group appears, with a possible contribution of ketenes.

- Stage 4: a large fraction of hydrogen has disappeared. $\mathrm{C}=0$ groups are degraded while acetylenic groups are still increasing while and $\mathrm{C}=\mathrm{C}$ groups are decreasing.

Raman measurements with a $532 \mathrm{~nm}$ excitation were unsuccessful due to a high fluorescence. Measurements collected with a $244 \mathrm{~nm}$ excitation reveal spectra with D and G bands, but the D band presents a substructure unusual for polyaromatic materials. These features may be the result of laser damaging, due to the fragility of the material. In any case, 
the behavior of PEG1450 under laser exposure is very different from what is-that of chondritic IOM. We also note that naked eye observations of the sample point to a yellow or brownish material, while polyaromatic materials are much darker. However, the final irradiated samples are consistent with a disordered cross-linked material based on Raman measurements and on its insolubility in methanol after high dose irradiation (Fig. A1).

\subsection{Irradiation of sucrose, lignin and cellulose with $17 \mathrm{MeV}$ Ni ions}

Sucrose, cellulose and lignin were prepared as thick pellets and analyzed ex situ by Raman spectroscopy. They were implanted with fluences of $1.510^{13}, 5.410^{13}$ and $710^{13}$ ions. $\mathrm{cm}^{-2}$, respectively (Tab. 1). No Raman spectra were collected with a $532 \mathrm{~nm}$ excitation on irradiated sucrose due to a high level of fluorescence, but the first-order carbon bands were obtained in the case of lignin and cellulose (Fig. 4). The spectra were decomposed into G and $\mathrm{D}$ bands with a double Gaussian fitting model (Tab. 3). The position of the G band (1567-1571 $\left.\mathrm{cm}^{-1}\right)$ and $\mathrm{I}_{\mathrm{D}} / \mathrm{I}_{\mathrm{G}}\left(\sim 0.8\right.$; Tab. 3) are consistent with an $\mathrm{sp}^{2}$-rich amorphous carbon film (Ferrari et al., 2001). The FWHM-G parameter is however smaller than in all amorphous samples of that study. These irradiated samples are definitely different from chondritic polyaromatic organic matter, which have a $\mathrm{sp}^{2}$ structure more similar to nano-crystalline graphite samples in Ferrari et al. (2001). Note however that a chemical gradient is expected as implanted swift ions have electronic interactions along their track as long as their energy is high enough so that $S_{e}>>S_{n}$, but eventually nuclear (i.e elastic) interactions are dominant at the end of the track (see section below).

\subsection{Low-energy irradiation with $40 \mathrm{keV}$ Carbon and $170 \mathrm{keV}$ Neon ions}

Thin films of PEG1450 as well as sucrose and cellulose pellets were irradiated with 40 $\mathrm{keV} \mathrm{C}$ and $170 \mathrm{keV} \mathrm{Ne}$ ions (Tab. 1). The penetration depth is very small (184 and $520 \mathrm{~nm}$, respectively) with respect to sample thickness, and no chemical evolution could be studied by transmission FTIR. $532 \mathrm{~nm}$ Raman spectroscopy probes the superficial thin layer, due to its dark color and to the resonant process that enhance the cross-section of the amorphous/disordered carbon with respect to the starting material. We observe that, above a critical nuclear dose, the sample is no longer fluorescent and first-order carbon bands appear (Fig. 5, 6, A3, A4). The bands are similar to those obtained in the sample implanted with 17 $\mathrm{Mev} \mathrm{Ni}$ ions, and they continuously evolve when increasing the nuclear dose. At a nuclear dose of about $60 \mathrm{eV}^{\text {.atom }}{ }^{-1}$, the $\mathrm{D}$ band is no longer distinguished from a broad pattern, and the Raman spectra are similar to those of completely hydrogen-free amorphous carbons (Fig. $5,6)$.

\subsection{Effect of temperature and air exposure}

Irradiations presented in this study were performed at room temperature, and may not be not relevant to cold astrophysical conditions. Since temperature effects have been reported earlier (Portugal et al., 2014), two experiments were run at $10 \mathrm{~K}$ on PEG 1450 with $0.76 \mathrm{MeV} / \mathrm{u}{ }^{78} \mathrm{Kr}$ ions (59 MeV). The quality of these data was not good enough to monitor accurately the chemical evolution of the sample, but they provide a qualitative assessment of temperature effect. We observe (Fig. A2) the production of volatile species as $\mathrm{CO}, \mathrm{CO}_{2}, \mathrm{CH}_{4}$ and $\mathrm{H}_{2} \mathrm{O}$ (or any other compound containing $-\mathrm{OH}$ ), which appear in the spectra as narrow features at 2139, 2339 and $3012 \mathrm{~cm}^{-1}$, and as a broad band around $3300 \mathrm{~cm}^{-1}$, respectively. These species sublimate after warming up the sample to room temperature. Besides the presence of these volatile species, the general evolution trend in 4 stages (Fig. 3) is also observed at $10 \mathrm{~K}$, and the spectra collected are overall fairly similar to those collected in room temperature experiments. An exception is the $-\mathrm{OH}$ band that displays several peaks at $10 \mathrm{~K}$ and which are still present after warming up. A possible explanation is that the water ice layer that condenses onto the sample during the experiment was reactive, possibly forming hydroxyl groups. We did not observe differences in kinetics as in Portugal et al., (2014), but our data 
did not allow accurate derivation of destruction/formation cross-sections. In any cases, the final products at similar doses were found similar.

Exposing irradiated samples to air may result in oxidation reactions with $\mathrm{O}_{2}$ or moisture, because they contain radicals (Yun et al., 2007). To address this issue, we measured the infrared spectrum of an irradiated PEG 1450 sample that was exposed to air and stored in dry and low- $\mathrm{O}_{2}$ conditions (Fig. A5). The exposition to air leads to a decrease of the aliphatic abundance, a slight drop of the peak at $\sim 1100 \mathrm{~cm}^{-1}$ and the disappearance of the $2200 \mathrm{~cm}^{-1}$ band due to the acetylenic group. Note that sample aging might have contributed to this chemical evolution, despite conservation in a desiccator maintained under primary vacuum with desiccant salts. Samples irradiated at low energy were transformed into amorphous carbons that resemble those observed in earlier studies, in particular studies based on in situ Raman analysis (Ferini et al., 2004). Altogether, the effect of air exposure is weak and does not blur the conclusions driven by ex-situ analyses.

\section{DISCUSSION}

\subsection{A critical amorphization dose}

The irradiation of PEG 1450 at high energy with $12 \mathrm{MeV}$ Carbon ions leads to the progressive structural evolution of the polymer towards a disordered structure, through dehydrogenation, chain breaking, cross-linking, the formation of carbonyl, methyl, olefinic and acetylenic functional groups or bonds. This evolution is typical of organic linear homopolymers and has been reported in many earlier studies (Balanzat et al., 1995). The electronic doses in our study are, however, higher than in previous studies $\left(0-32\right.$ eV.atom ${ }^{-1}$; 1.7-457 MGray) and the final irradiated sample is dramatically modified. It is now interesting to have a comparison with carbonaceous reference samples usually used in material sciences. Disordered and amorphous carbon materials have been classified into different groups based on their $\mathrm{sp}^{2} / \mathrm{sp}^{3}$ ratio, $\mathrm{H} / \mathrm{C}$ ratio, optical gap and their degree of order/disorder according to their Raman spectra (Ferrari and Robertson, 2000, 2001). This classification relies on endmembers: a-C (pure carbon, $\mathrm{sp}^{2}$-rich), ta-C (pure carbon, $\mathrm{sp}^{3}$-rich), a-C:H (hydrogenated, $\mathrm{sp}^{2}$ rich), ta-C:H (hydrogenated, $\mathrm{sp}^{3}-\mathrm{rich}$ ), nc-G (nano-crystalline graphite) and polymeric hydrogen-rich a-C:H or ta- $\mathrm{C}: \mathrm{H}$ films. In practice, the Raman spectra collected with different excitation wavelengths of these proxies are compared to the sample, providing clues on its $\mathrm{sp}^{2}$ structure. It is worth mentioning that the similarity between Raman spectra does not mean materials are similar: for instance, ligno-cellulosic coals and chondritic IOM fits with nanographitic carbon, but they are of course different in terms of composition and structure. Here, irradiated PEG films with heavy swift ions resemble to polymeric carbon films, i.e. a H/C ratio and a macromolecular structure, leading to a high fluorescence with visible laser excitations (Ferrari and Robertson, 2001). In contrast, low energy irradiations (40 keV C and $170 \mathrm{keV} \mathrm{Ne}$ ) show a progressive broadening of the $\mathrm{G}$ band $\left(\sim 115\right.$ to $\left.173 \mathrm{~cm}^{-1}\right)$ and the shortwards shift of its peak position, from $\sim 1570$ to $1545 \mathrm{~cm}^{-1}$ (Fig. 5, A3; Tab. 3) with ID/IG higher than 0.6. These values are consistent with the transformation into a predominantly $\mathrm{sp}^{2}$-rich amorphous carbon and with the dark color of the films. Upon increasing the nuclear dose, the decrease of the G-band position suggests an increase of $\mathrm{sp}^{3}$ carbon atoms, which also corresponds to an increase in the degree of disorder. Besides, we observe a slight precursor effect, the PEG sample reaching the highest degree of disorder. The $244 \mathrm{~nm}$ Raman spectrum collected for the irradiated PEG sample discards the presence of ta-C and polymeric a-C:H. In all cases, the irradiated samples do not fit the composition and the structure of chondritic IOM, based on infrared and Raman spectra, respectively (Fig. 8, 9).

In order to disentangle the respective effects of electronic and nuclear interactions, we have compared these results with earlier studies that have characterized irradiated samples by Raman spectroscopy. Table 4 compiles the targets and experimental conditions of those studies, as the nature and energy of the ions, the stopping powers and maximum fluences. The

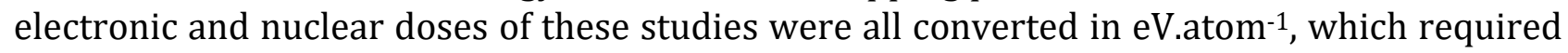
some assumption on the nature of the target in some cases. These values should be considered as crude estimates (as to within $\sim 20 \%$ ), because the electronic and nuclear 
stopping powers depend on the target. Figure 7 reports the width of the $G$ band plotted versus its peak position (the broader D-band was not considered, due to less precision in its peak position and width, which are very sensitive to the fitting model). As already observed (Baratta et al., 2004; Brunetto et al., 2009), samples irradiated with low nuclear doses are located at high Raman shift and small width (zone 1), while those irradiated with a high dose are located at low Raman shift and large width (zone 2). The frontier between these two zones is here set to FWHM-G $=120 \mathrm{~cm}^{-1}$ and $\omega_{\mathrm{G}}=1570 \mathrm{~cm}^{-1}$, which corresponds to the reference a-C carbon film abovementioned. Plotting the width and peak position of the $G$ band of all data, from literature and those of this study, reveals a transition at $10_{-7}^{+10} \mathrm{eV} \mathrm{atom}^{-1}$, which furthermore seems weakly sensitive to the nature of the precursor (Fig. 7). Below $10_{-7}^{+10}$ eV.atom ${ }^{-1}$, the G-band width is in the range $40-120 \mathrm{~cm}^{-1}$ and its peak position is $>1570 \mathrm{~cm}^{-1}$ (zone 1). Beyond this dose, the G-band width is larger than $120 \mathrm{~cm}^{-1}$ and moves to lower Raman shifts between $1530-1570 \mathrm{~cm}^{-1}$ (zone 2). Additional irradiations, up to nuclear doses greater than $100 \mathrm{eV}^{\text {atom }}{ }^{-1}$, still generate an amorphous carbon, with possibly an increase of $\mathrm{sp}^{3}$ carbon atoms. Some carbonaceous precursors do not display the first-order carbon bands before irradiation, such as ice mixtures, photolytic refractory residues, the polyimide Kapton $\mathrm{H}$ polymer and the precursors used in this study. Two cases are here observed concerning the appearance of the $\mathrm{D}$ and $\mathrm{G}$ bands. At low energy (elastic interactions) and for doses greater than $10_{-7}^{+10} \mathrm{eV}_{\text {.atom }}{ }^{-1}$, the parameters of the G-band directly locate in zone 2 . In the case of swift heavy ions and electronic interactions (sample thickness $<<$ implantation length), the final structure strongly depends on the composition of the target. In the case of the PEG1450 polymer, these bands never arise, and the irradiated sample remains fluorescent. In the case of the polyimide polymer, which contains three aromatic rings in its monomer, a polyaromatic structure appears at low nuclear dose $\left(0.1 \mathrm{eV}^{-a_{0} \mathrm{t}^{-1}}{ }^{-}\right.$Costantini et al., 2002). The G-band width remains lower than $120 \mathrm{~cm}^{-1}$ and the peak position ranges between 1577 and $1587 \mathrm{~cm}^{-}$ 1 , which is consistent with zone 1 . Besides, this shows that this irradiated sample is structurally similar to chondritic IOM or to nano-graphitic samples, based on their respective Raman spectra (Fig. 9).

The nuclear dose of $10_{-7}^{+10} \mathrm{eV}^{-a_{0} \mathrm{~m}^{-1}}$ appears as the threshold energy triggering the structural transformation of any carbonaceous precursor into a $\mathrm{sp}^{2}$-rich amorphous carbon. Carbonaceous materials can receive much higher electronic doses without having their structure strongly modified, as in the case of polyimide irradiated with an electronic dose up to $1070 \mathrm{eV}$. atom $^{-1}$ (Costantini et al., 2002b). Indeed, the creation of atomic vacancies is mostly controlled by nuclear interactions, easing chain splitting and cross-linking by atomic displacements. In a perfect crystal, the minimum energy to displace an atom to a near interstitial site is typically $5-10 \mathrm{eV}$. The energy that an atom must receive to be moved permanently to a distant interstitial site is therefore higher. This energy, named the displacement energy $E_{d}$, is that an atom receives from a single collision from the incoming ion. It depends essentially on the atom mass, temperature, the composition and structure of the target, but little on the nature of the incident ion. For a carbon atom, $E_{d}$ is $35 \mathrm{eV}$ in the case of diamond, $10-20 \mathrm{eV}$ in the case of graphite and $\sim 10 \mathrm{eV}$ for amorphous carbons (McKenna et al., 2016; Nakai et al., 1991). The TRIM software uses an average value of $28 \mathrm{eV}$. Because $\mathrm{sp}^{3}$ carbons in diamond are more resistant to irradiation than $\mathrm{sp}^{2}$ carbons, the value is higher in the case of diamond. For graphite, the cristallographic anisotropy leads to a higher value of $E_{d}$ when irradiating in the plane of the graphene sheets. This explains the range of values reported in the literature, which are higher than that of amorphous carbon. Carbonaceous materials formed by irradiation get close to the amorphous carbons abovementioned, thus the displacement energy lies around $10 \mathrm{eV}$. Altogether, the critical nuclear dose of $10_{-7}^{+10} \mathrm{eV}^{-a t o m}$ 1 reflects the energy that carbon atoms need to be displaced to a new interstitial site $(\sim 1$ displ.atom ${ }^{-1}$ ). The sharpness of the threshold shows that for all the precursors considered here, either from the present study or studies published earlier, the carbon displacement energies are similar whatever the starting material.

The case of sucrose, lignin and cellulose implanted by swift heavy $10 \mathrm{MeV} \mathrm{Ni}$ ions reflects the combination of two mechanisms. As mentioned above, the interactions between 
the ion and the target are essentially electronic, except at the end of the track where the low energy of the ion results in elastic interactions. The $G$ band of irradiated lignin and cellulose samples lies at the edge of the boundary of zones 1 and 2. It is consistent with an amorphous carbon zone localized around the implantation depth, whose formation is triggered by a $10_{-7}^{+10} \mathrm{eV}$.atom ${ }^{-1}$ nuclear dose. As mentioned before, the G-band fits with low-energy irradiated samples with the lowest nuclear dose. Above the implantation zone, the material is chemically modified but still displays Raman properties of a polymeric amorphous carbon. Note that despite the fact that lignin contains phenols, no polyaromatic structure was formed and the signature of the amorphous carbon dominates the Raman spectrum at the end of the track.

\subsection{Origin of organic matter in chondritic cosmomaterials}

We discuss now the pro and cons of a formation scenario of chondritic organic matter by the radiolysis of interstellar precursors. Two primary sources of radiation must be examined: solar energetic particles (stellar wind, flares) and galactic cosmic rays (GCR). We consider interstellar precursors as submicrometer-sized grains, in agreement with both the typical size of organic matter particles observed in the matrix of primitive chondrites and dust size estimations in the interstellar medium (Changela, 2015; Le Guillou et al., 2014; Mathis et al., 1977). Testing an interstellar heritage, i.e. no significant contribution of grains formed in the presolar disk, we assume those grains were partly constituted by the carrier of the socalled Aromatic Interstellar Bands (AIBs), i.e. Polycyclic Aromatic Hydrocarbon molecular entities made of 20-100 atoms (Foschino et al., 2019). These species are larger than the polyaromatic units detected in chondritic IOM (1-4 rings, 6-20 atoms) (Cody et al., 2002; Derenne and Robert, 2010) and they are devoid of oxygen, nitrogen or sulfur. But a source of heteroatoms could be represented by ices, which are, in molecular clouds, predominantly composed of $\mathrm{H}_{2} \mathrm{O}, \mathrm{CO}, \mathrm{CO}_{2}, \mathrm{CH}_{3} \mathrm{OH}, \mathrm{H}_{2} \mathrm{CO}, \mathrm{NH}_{3}, \mathrm{HCN}$ and include sulfur compounds (Boogert et al., 2015).

We first estimate the nuclear and electronic doses deposited by GCR in grains during $20 \mathrm{Myr}$, in local ISM before the collapse of the molecular cloud, or in the low gas density regions of the proto-solar disk $\left(<10^{4} \mathrm{~cm}^{-3}\right)$. The size of the grains $(<100 \mathrm{~nm})$ is low enough to prevent a significant contribution of cosmic-ray particles to be implanted into the grain. In the case of $\mathrm{Fe}$, for instance, the ions that are stopped by a $100 \mathrm{~nm}$ grain $(\mathrm{E}<0.075 \mathrm{MeV})$ provide an electronic dose that is $3 \times 10^{7}$ lower than all other ions. The electronic and nuclear stopping powers, $S_{e}$ and $S_{n}$, are calculated with the SRIM software for a material with a composition $\mathrm{H}: \mathrm{C}: \mathrm{O}=1: 1: 0.2$, a density of $1.5 \mathrm{~g} . \mathrm{cm}^{-3}$, and a mean molar mass for each atom of $(12+1+16 \times 0.2) / 2.2=7.4 \mathrm{~g} \mathrm{~mol}^{-1}$. This material is a rough analog of a mixture of polyaromatic carbonaceous dust mixed with water ice. The GCR flux density for protons is taken from Webber and Yushak (1983):

$$
\frac{d N}{d E}=\frac{C E^{0.3}}{\left(E+E_{0}\right)^{0.3}} \quad \text { particles. } \mathrm{cm}^{-2} \cdot s r^{-1}\left(\mathrm{MeV} \cdot n u c^{-1}\right)^{-1}
$$

where $\mathrm{E}$ is the energy per nucleon (nuc), and $\mathrm{C}=9.42 \times 10^{4}$ is a normalisation constant. We use

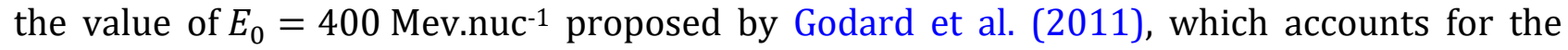
typical ionization rate measured in molecular clouds. We note that more recent parametrisations of the interstellar flux are available in the literature, e.g. Ghelfi et al., (2016), but the simple form used here is sufficient for our purpose (cosmic abundances are another important source of uncertainties for heavy species). The contribution of an ion with $\mathrm{Z}>1$ is calculated as the proton flux density multiplied by its cosmic abundance taken from Meyer et al. (1998). The dose accumulated in one single grain can then be written: 


$$
\text { Dose }\left[e V \cdot a t^{-1}\right]=\frac{4 \pi M}{\rho N_{A}} \sum_{Z=1}^{92} \int_{E_{\min }}^{E_{\max }}\left(\frac{d N}{d E}\right) S_{e, n}^{Z} d E
$$

with $\rho=1.5$ g.cm ${ }^{-3}, M=7.4$ g.mol-1, $\mathrm{N}_{A}=6.02 \times 10^{23}$. In practice, the summation runs over the most abundant elements $\mathrm{H}, \mathrm{He}, \mathrm{C}, \mathrm{O}, \mathrm{Mg}, \mathrm{Al}, \mathrm{Si}, \mathrm{S}$. and $\mathrm{Fe}$. $\mathrm{E}_{\min }$ and $\mathrm{E}_{\max }$ are set to 0.1/A and 10000 /A MeV.nuc ${ }^{-1}$, respectively, with A the nuclear mass.

Our calculations show that for a duration of 1-20 Myr, the electronic dose accumulated by a single grain ranges is within the range of 0.085 and $1.7 \mathrm{eV}$.atom ${ }^{-1}$ (Tab. 5). The nuclear dose is extremely low $\left(<8.8 \times 10^{-4} \mathrm{eV}\right.$ atom $\left.{ }^{-1}\right)$, i.e. orders of magnitude lower than the critical dose of $10_{-7}^{+10} \mathrm{eV}$.atom ${ }^{-1}$ reported above.

The role of particles emitted by the protosun is more difficult to assess. The activity of the early sun can be inferred from T-Tauri star observations, and the X-ray-EUV emission and stellar wind are usually estimated to be 1000 times more active than the present sun (Ribas et al., 2005). However, in a T-Tauri disk the protostar is surrounded by a dense dusty gas $\left(\sim 10^{12}\right.$ $\mathrm{cm}^{-3}$, Visser et al. (2018)), which slows down solar particles. For $\mathrm{n}_{2}=10^{12} \mathrm{~cm}^{-3}, 10 \mathrm{MeV}$ protons are stopped at $0.001 \mathrm{AU}$, which means that no significant nuclear or electronic dose is deposited in grains outside this tiny region. As a result, only the contribution of GCR needs to be considered. These results are consistent with the upper values of 0.5 and $0.2 \pm 0.1$ displ.atom $^{-1}$

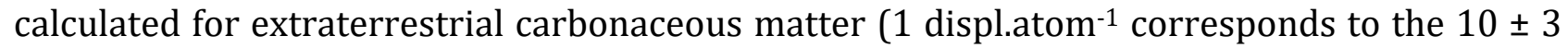
eV.atom ${ }^{-1}$ critical nuclear dose) (Baratta et al., 2004; Brunetto et al., 2009).

The irradiation of PEG1450 with $12 \mathrm{MeV}$ Carbon ions shows dehydrogenation and carbonization processes. At a dose of $32 \mathrm{eV} . a t o m^{-1}$, the solid residue is disordered, highly cross-linked, and shares similarities with IOM composition of the most primitive CR chondrites (Abreu and Brearley, 2010) (Fig. 8). The alkyl band is similar in terms of the intensity ratio of the $\mathrm{CH}_{2}$ and $\mathrm{CH}_{3}$ anti-symmetric stretching components, and points to short alkyl chains. The relative intensity of the aliphatic and carbonyl bands to the $\mathrm{C}=\mathrm{C}$ band are close to those in IOM. However, unsaturated carbons are mostly olefinic and acetylenic, while they are aromatic in IOM. This observation shows that no aromatic species are synthesized during irradiation. In contrast, earlier studies have shown that aromatic-rich precursors can transform into polyaromatic compounds: for instance, this is the case of Kapton $\mathrm{H}$ exposed to swift heavy ions (Costantini et al., 2002b; Severin et al., 2005, 2010). Polyimide Kapton H (pyromellitic dianhydride-oxydianiline) is a polymer based on a monomer formed by 3 aromatic rings and two five atom rings. Its irradiation by swift heavy ions leads to semiconducting polyaromatic carbon films. Raman spectroscopy investigations show a progressive evolution through the appearance of $\mathrm{G}$ and $\mathrm{D}$ bands, whose spectral properties fit those of IOM, in particular the peak position and width of the G-band, and the ratio of peak intensities $\mathrm{I}_{\mathrm{D}} / \mathrm{I}_{\mathrm{G}}$ (Costantini et al., 2002b; Quirico et al., 2014).

In the case of ice and PAHs mixtures, the irradiation process needs to fulfill two main conditions: (i) disrupt PAHs into 1-4 rings polyaromatic units and bond them with an aliphatic linkage made of short alkyl chains, and (ii) incorporate $\mathrm{O}, \mathrm{N}$ and $\mathrm{S}$ heteroatoms within the structure. Regarding the first point, ion irradiation of graphite, graphene and carbon nanotubes with heavy swift ions generates defects in the planar hexagonal lattice of carbon sheets (Olejniczak and Skuratov, 2014; Zhai et al., 2014). In graphite and nanotubes, for instance, a D band arises and grows continuously with an increasing dose. Such structural modifications suggest that large PAH entities may be disrupted into smaller ones. Regarding the second point, irradiation of mixtures of water ice with hydrogenated amorphous carbon shows that oxygen recombines with carbon atoms to form $\mathrm{CO}_{2}$ molecules (Mennella et al., 2004). Proton irradiation of isolated coronene in water ice leads to the addition of side chains containing oxygen, but also to the conversion of aromatic to aliphatic carbons by hydrogenation at the edge of coronene molecules (Bernstein et al., 2003). This study does not report the fragmentation of coronene, despite an electronic dose of $\sim 2$ eV.atom ${ }^{-1}$ was delivered. However, the dose is not a good tracer of the chemical mechanisms (Bouffard et al., 1995) and the radiolytic cross-sections (here a destruction cross-section) follow a power law 
with the stopping power Se (Godard et al., 2011; Seperuelo Duarte et al., 2010). A comprehensive approach of this issue should rely on experiments spanning a broad range of ions/stopping powers, including swift heavy ions.

Finally, a formation mechanism of chondritic organic matter from mixtures of PAHs and simple ices appears plausible and would reconcile the coexistence in chondritic IOM of polyaromatic species formed at high temperature, with deuterium hot spots that require very low temperature conditions ( $<20 \mathrm{~K}$ ) (Parise et al., 2006; Roberts and Millar, 2000; Watanabe and Kouchi, 2008). This scenario would also account for the isotopic heterogeneities $\delta^{13} \mathrm{C}, \delta$ ${ }^{15} \mathrm{~N}$ and $\delta \mathrm{D}$ at the molecular scale. Hence, Okumura and Mimura (2011) report that the aliphatic linkage of IOM from the Murchison (CM2) chondrite are D and ${ }^{13} \mathrm{C}$-rich, while the thermally stable polyaromatic component is $\mathrm{D}$ and ${ }^{13} \mathrm{C}$-depleted. They conclude that the thermally labile component is composed of ISM polar compounds, while the second is made of ISM PAHs. Obviously, this view needs to be supported by dedicated experiments on targets including both PAHs and simple ices.

\subsection{Ion implantation in planetary surfaces}

The cold surfaces of Trans-Neptunian Objects (TNOs), Centaurs, Pluto and Charon north polar cap contain reddish organic materials (Brown, 2012; Doressoundiram et al.; Grundy et al., 2016, 2018; Lacerda et al., 2014, 2014; Merlin et al., 2017; Schmitt et al., 2017; Stern et al., 2015). Their origin is debated and three main scenarios have been proposed: (i) synthesis in the protosolar disk or the planetary body (Sekine et al., 2017), (ii) radiolysis of simple ices (Brown, 2012; Cooper et al., 2003) and (iii) sedimentation of atmospheric aerosols in the case of Pluto (Grundy et al., 2018).

TNOs surfaces have been continuously exposed to Solar wind and GCRs for 4.55 Gyr, resulting in high nuclear and electronic doses, deposited at the surface and in the subsurface down to several meters. Low energy ions from Solar wind have a shallow penetration depth $(<1 \mu \mathrm{m})$ and experience strong elastic interactions with surface materials. In contrast, the high energy ( $>1 \mathrm{Mev} / \mathrm{nuc}$ ) component of GCRs deeply penetrates and energy is essentially deposited through electronic interactions, except where ions eventually implant. We first examine the conditions for which organic components at the surface are transformed into a

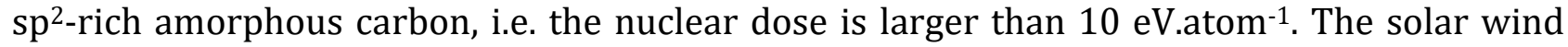
fluxes of the $\mathrm{H}, \mathrm{He}, \mathrm{C}, \mathrm{O}, \mathrm{Mg}, \mathrm{Si}, \mathrm{S}$ and Fe elements were calculated from the He fluence of Mewaldt et al. (2007) using the solar abundances of Asplund et al. (2009). The solar wind flux $F(D)$ at an heliocentric distance $D$ is assumed to equal $F(D=1 A U) / D^{2}$. The stopping powers are calculated with the SRIM software for a PEG material (see section 4.2). The contribution of Solar Energetic Particles (SEP) has not been implemented as did Urso et al. (2020), because the flux of Mewaldt et al. (2007a) covers the full spectral range of solar wind, including the suprathermal tail whose flux intensity is higher than that of SEPs (Mewaldt et al., 2007b).

Over long time scales, sputtering is an issue and has been evaluated for $\mathrm{H}, \mathrm{He}, \mathrm{C}, \mathrm{S}$ and Fe. Sputtering yield values of carbonaceous materials are very scarce in literature, and we

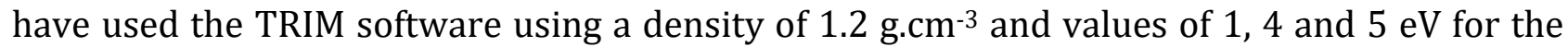
lattice and surface binding energies and the displacement energy (Stanishevsky, 2001; Ullmann et al., 1993; Vuković et al., 2018), respectively. As only few values of these parameters were found in literature, we have numerically tested their effect on the sputtering yield. Finally, upper values of the sputtering yields $\mathrm{Y}_{\mathrm{i}}(\mathrm{E}) \mathrm{i}=\mathrm{H}, \mathrm{C}, \mathrm{O}, \mathrm{Mg}, \mathrm{Si}, \mathrm{S}, \mathrm{Fe}$ ) over the range $1 \mathrm{keV}-1 \mathrm{MeV}$ were selected upon varying energy and the abovementioned parameters, to estimate the sputtered thicknesses of each element at a given heliocentric distance D. These calculations show that He is the main contributor to sputtering, and we found erosion depth Ds of $24 \mu \mathrm{m}, 2 \mu \mathrm{m}$ and $20 \mathrm{~nm}$ at 30, 100 and $1000 \mathrm{AU}$, respectively. Sputtering and implantation reach equilibrium and the actual dose deposited in the uppermost surface corresponds to an actual irradiation time that can be roughly estimated to $T_{\text {real }}=$ $4.5510^{9} D_{S} / \bar{D}_{I}(\mathrm{He})$ years, where $\bar{D}_{I}(\mathrm{He})$ is the penetration depth of the He ion averaged over the solar wind flux between $2 \times 10^{-4}$ and $1 \mathrm{Mev} /$ nuc. The results are presented in Table $6 . \mathrm{H}$ and $\mathrm{He}$ are the main contributors to the nuclear dose, other elements generate a nuclear dose 
lower than 10 eV.atom- ${ }^{-1}$. At 30, 100 and $1000 \mathrm{AU}$, the nuclear doses deposited by $\mathrm{H}$ and $\mathrm{He}$ are larger than $10 \mathrm{eV}$.atom ${ }^{-1}$, which means that any organic material at the surface of the TNO would be transformed into an amorphous carbon. The amorphous carbon layer is very thin, as $\bar{D}_{I}(\mathrm{He})=67 \mathrm{~nm}$ and $\bar{D}_{I}(H)=31 \mathrm{~nm}$. It is noteworthy that this calculation is very rough: it does provide evidence that amorphous carbon is generated, but its spatial distribution upon depth remains to be determined. The contribution of GCR to TNOs irradiation requires the actual GCRs flux in the outer Solar System (Cooper et al., 2003). According to Usoskin et al. (2005), the flux density at $30 \mathrm{AU}$ is lower by a factor 1.6 at $1 \mathrm{MeV} /$ nucleon and 5 at 0.1 $\mathrm{MeV} /$ nucleon, with respect to Webber and Yushak (1983). This range spans around the Braag peak of the electronic stopping power, and account for a large fraction of the energy deposited by electronic interactions. In contrast, the flux is much weaker in the range $10 \mathrm{keV}-100 \mathrm{keV}$, which corresponds to the nuclear stopping power. Therefore, the nuclear dose calculated using the flux density of Webber and Yushak (1983) will be overestimated. For the uppermost

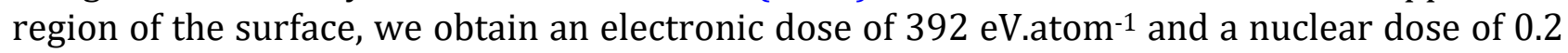
eV.atom ${ }^{-1}$. The nuclear dose does not allow a direct amorphization, but the electronic dose is high and leads to a significant carbonization of the material (see the high energy irradiation of PEG 1450 above).

Due to their low energy, solar ions have a low penetration depth (mean $\sim 70 \mathrm{~nm}$ ). In contrast, GCRs penetrate much deeper, down to several meters for the highest energetic ions. As a consequence, we expect solar wind to essentially control the visible range of VNIR reflectance spectra, while the effect of GCRs could be detected in the NIR range up to $2.5 \mu \mathrm{m}$. Solar wind generates amorphous carbon materials as demonstrated by our low energy data. In the case of GCRs, the nuclear dose is $<0.2 \mathrm{eV}_{\text {.atom }}^{-1}$ along the ion track but it dramatically increases when the ion implants and amorphous carbon is formed.

Raman spectroscopy shows that amorphous carbons are $\mathrm{sp}^{2}$-rich, resembling to a-C carbon films, with a varying degree of disorder in the case of low-energy irradiations. There is a dramatic evolution of the composition and structure of the precursor, with little precursor effects. The exact nature of the irradiated material is, however, not fully elucidated. Raman spectroscopy only reveals the $\mathrm{sp}^{2}$-structure and no infrared spectra could be collected on these very thin films. The predominance of $\mathrm{sp}^{2}$ carbons should be considered as robust, consistent with the strong visible absorption of the samples, but they are not similar to the carbon films used as references in the Raman study. We reasonably infer a significant dehydrogenation followed by the formation of sp2 rings, but 0 atoms likely remain in the structure. Nevertheless, the $\mathrm{sp}^{2}$ structure of carbonaceous materials control the optical constants in the UV-Vis (Applin et al., 2018; Hendrix et al., 2016; Robertson, 1995), and clues on the most suitable optical constants to simulate surface darkening and VNIR spectra with Hapke models can be obtained (Merlin et al., 2007). Optical constants published in Zubko et al. (1996) have been widely used for this purpose and consist of three types of carbon films: a sputtered a-C (ACAR); a $\mathrm{sp}^{3}$-rich hydrogenated amorphous carbon (ACH2) and soots produced from benzene combustion (BE). ACAR and BE display a strong absorption in the UV, visible and infrared ranges (10-0.1 eV), while ACH2 shows strong absorption in UV, moderate and reddish in the visible and weak in the near-infrared (see Figure 2 in Zubko et al. (1996) for details). ACH2 is definitely inconsistent with the amorphous carbon identified in our irradiated samples, according to the Raman characterization. BE is soot and is structurally very different from an amorphous sample, as it is composed of large polyaromatic units (Brunetto et al., 2009). Finally, ACAR appears as the closest analogue to the $\mathrm{sp}^{2}$-rich amorphous carbon produced by irradiation and should be preferred for modeling reflectance VNIR observations of dark small bodies. In the future, the optical constants of irradiated films should be measured.

At last, the question arises whether the formation of amorphous carbon can be detected in VNIR spectra. According to our calculations, SW irradiation leads to a very thin film ( $\sim 70 \mathrm{~nm}$ in average). An optical path of $140 \mathrm{~nm}$ generates a transmission of only 0.02 at $0.4 \mu \mathrm{m}$ and 0.2 at $0.8 \mu \mathrm{m}$, according to the optical constants of Zubko et al. (1996). These calculations are consistent with experiments and modeling of snow reflectance contaminated 
by $2.5 \mathrm{ppm}$ of black carbons which show that such a low amount is enough to decrease the reflectance by 15\% (Brandt et al., 2011; Dang et al., 2015). A darkening effect is then detectable in the visible range, with possibly some reddening. The darkening may even be stronger, because, as we mentioned before, we have only considered the mean depth. The nuclear dose may exceed the $10 \mathrm{eV}^{-a_{0} \mathrm{~m}^{-1}}$ value deeper. More detailed calculations are required here to go further.

\section{CONCLUSION}

We report the results of low and high-energy ion irradiation experiments of a variety of macromolecular organic materials. The main conclusions of our study are the following:

- In the case of low energy irradiation that favors elastic interaction between ion and target, i.e. solar wind irradiation or GCRs implantation, a critical nuclear dose of about 10 eV.atom ${ }^{-1}$ controls the transformation of the starting organic material into an amorphous carbon that shares similarities with $\mathrm{sp}^{2}$-rich a-C amorphous carbons.

- In a range of heliocentric distances ranging between 30 and 1000 AU, organic materials at the surface of TNOs are transformed into an $\mathrm{sp}^{2}$-rich amorphous carbon by Solar wind in the first tens of nanometers. This process generates darkening, and possibly reddening, in the visible range of VNIR reflectance spectra. The optical constants of the amorphous carbon ACAR from Zubko et al. (1996) should be suitable to simulate VNIR spectra. GCRs penetrate more in-depth in the surface, generating intense chemistry, and may generate "amorphous carbon" as well.

- The effect of high energy swift ions in the electronic regime deposition triggers radiolytic carbonization of irradiated organic components, including dehydrogenation, the formation of olefinic and acetylenic bonds, cross-linking and a final disordered structure. No aromatic species are produced. As a consequence, chondritic IOM is not formed from the radiolysis of simple ices or simple organics.

- In contrast, a plausible scenario is a formation through GCR irradiation of submicrometric grains containing PAHs and simple ice mixtures, which may generate a polyaromatic structure with heteroatoms and short aliphatic chains. 


\section{ACKNOWLEDGEMENTS}

We are grateful to the GANIL facility for high energy irradiation experiments; Jean Duprat and CSNSM for low energy irradiation experiments (beamlines Sidonie and Irma), and to the Raman National facility at Laboratoire de Géologie/ENS-Lyon. This project has been funded by a grant from University Grenoble Alpes (program AGIR) and the Centre National d'Etudes Spatiales (CNES). We warmly thank G. Strazzulla for earlier discussions on this project, and two anonymous reviewers for their comments and suggestions that improved the manuscript. 


\section{REFERENCES}

Abreu, N.M., and Brearley, A.J. (2010). Early solar system processes recorded in the matrices of two highly pristine CR3 carbonaceous chondrites, MET 00426 and QUE 99177. Geochim. Cosmochim. Acta 74, 1146-1171.

Alexander, C.M.O., Russell, S.S., Arden, J.W., Ash, R.D., Grady, M.M., and Pillinnger, C.T. (1998). Alexander98_MPS_33_603.pdf. Meteorit. Planet. Sci. 33, 603-622.

Alexander, C.M.O., Fogel, M., Yabuta, H., and Cody, G.D. (2007). The origin and evolution of chondrites recorded in the elemental and isotopic compositions of their macromolecular organic matter. Geochim. Cosmochim. Acta 71, 4380-4403.

Alexander, C.M.O., Cody, G.D., De Gregorio, B.T., Nittler, L.R., and Stroud, R.M. (2017). The nature, origin and modification of insoluble organic matter in chondrites, the major source of Earth's C and N. Chem. Erde - Geochem. 77, 227-256.

Applin, D.M., Izawa, M.R.M., Cloutis, E.A., Gillis-Davis, J.J., Pitman, K.M., Roush, T.L., Hendrix, A.R., and Lucey, P.G. (2018). Ultraviolet spectral reflectance of carbonaceous materials. Icarus 307, 40-82.

Asplund, M., Grevesse, N., Sauval, A.J., and Scott, P. (2009). The chemical composition of the Sun. Annu. Rev. Astron. Astrophys. 47, 481-522.

Auger, A.-T., Groussin, O., Jorda, L., Bouley, S., Gaskell, R., Lamy, P.L., Capanna, C., Thomas, N., Pommerol, A., Sierks, H., et al. (2015). Geomorphology of the Imhotep region on comet 67P/Churyumov-Gerasimenko from OSIRIS observations. Astron. Astrophys. 583, A35.

Balanzat, E., Betz, N., and Bouffard, S. (1995). Swift heavy ions modification of polymers. 105, 46-54.

Baratta, G.A., Mennella, V., Brucato, J.R., Colangeli, L., Leto, G., Palumbo, M.E., and Strazzulla, G. (2004). Raman spectroscopy of ion-irradiated interplanetary carbon dust analogues. J. Raman Spectrosc. 35, 487-496.

Bernard, S., Beyssac, O., Benzerara, K., Findling, N., Tzvetkov, G., and Brown, G.E. (2010). XANES, Raman and XRD study of anthracene-based cokes and saccharose-based chars submitted to high-temperature pyrolysis. Carbon 48, 2506-2516.

Bernstein, M.P., Moore, M.H., Elsila, J.E., Sandford, S.A., Allamandola, L.J., and Zare, R.N. (2003). Side Group Addition to the Polycyclic Aromatic Hydrocarbon Coronene by Proton Irradiation in Cosmic Ice Analogs. Astrophys. J. 582, L25-L29.

Beyssac, O., Brunet, F., Petitet, J.-P., Goffé, B., and Rouzaud, J.-N. (2004). Experimental study of the microtextural and structural transformations of carbonaceous materials under pressure and temperature. Eur. J. Mineral. 15, 937-951.

Binet, L., Gourier, D., Derenne, S., and Robert, F. (2002). Heterogeneous distribution of paramagnetic radicals in insoluble organic matter from the Orgueil and Murchison meteorites. Geochim. Cosmochim. Acta 66, 4177-4186.

Binet, L., Gourier, D., Derenne, S., Robert, F., and Ciofini, I. (2004). Occurence of abundant diradicaloid moieties in the insoluble organic matter from the Orgueil and Murchison meteorites: a fingerprint of its extraterrestrial origin? Geochim. Cosmochim. Acta 68, 881891.

Boogert, A.C.A., Gerakines, P.A., and Whittet, D.C.B. (2015). Observations of the Icy Universe. Annu. Rev. Astron. Astrophys. 53, 541-581.

Bouffard, S., Gervais, B., and Leroy, C. (1995). Basic phenomena induced by swift heavy ions in polymers. Nucl. Instrum. Methods Phys. Res. Sect. B Beam Interact. Mater. At. 105, 1-4.

Brandt, R.E., Warren, S.G., and Clarke, A.D. (2011). A controlled snowmaking experiment testing the relation between black carbon content and reduction of snow albedo. J. Geophys. Res. 116, D08109.

Brown, M.E. (2012). The Compositions of Kuiper Belt Objects. Annu. Rev. Earth Planet. Sci. 40, 467-494.

Brunetto, R., Pino, T., Dartois, E., Cao, A.-T., d'Hendecourt, L., Strazzulla, G., and Bréchignac, Ph. (2009). Comparison of the Raman spectra of ion irradiated soot and collected extraterrestrial carbon. Icarus 200, 323-337. 
Burton, A.S., Stern, J.C., Elsila, J.E., Glavin, D.P., and Dworkin, J.P. (2012). Understanding prebiotic chemistry through the analysis of extraterrestrial amino acids and nucleobases in meteorites. Chem. Soc. Rev. 41, 5459.

Busemann, H., Young, A.F., Alexander, C.M.O., Hoppe, P., Mukhopadhyay, S., and Nittler, N. (2006). Interstellar Chemistry Recorded in Organic Matter from Primitive Meteorites. Science $312,727-730$.

Changela, H.G. (2015). Morphological study of Insoluble Organic Matter from carbonaceous chondrites: Correlation with petrologic grade. Geochim. Cosmochim. Acta 159, 285-297.

Cleeves, L.I., Bergin, E.A., Alexander, C.M.O., Du, F., Graninger, D., Oberg, K.I., and Harries, T.J. (2014). The ancient heritage of water ice in the solar system. Science 345, 1590-1593.

Cody, G.D., and Alexander, C.M.O. 'D. (2005). NMR studies of chemical structural variation of insoluble organic matter from different carbonaceous chondrite groups. Geochim. Cosmochim. Acta 69, 1085-1097.

Cody, G.D., Alexander, C.M.O., and Tera, F. (2002). Solid-state ( $1 \mathrm{H}$ and 13 C) nuclear magnetic resonance spectroscopy of insoluble organic residue in the Murchison meteorite: a selfconsistent quantitative analysis. Geochim. Cosmochim. Acta 66, 1851-1865.

Cody, G.D., Heying, E., Alexander, C.M.O., Nittler, L.R., Kilcoyne, A.L.D., Sandford, S.A., and Stroud, R.M. (2011). Establishing a molecular relationship between chondritic and cometary organic solids. Proc. Natl. Acad. Sci. 108, 19171-19176.

Cooper, P.D., Johnson, R.E., and Quickenden, T.I. (2003). Hydrogen peroxide dimers and the production of 02 in icy satellite surfaces. Icarus 166, 444-446.

Costantini, J.-M., Couvreur, F., Salvetat, J.-P., and Bouffard, S. (2002a). Micro-Raman study of the carbonization of polyimide induced by swift heavy ion irradiations. Nucl. Instrum. Methods Phys. Res. Sect. B Beam Interact. Mater. At. 194, 132-140.

Costantini, J.-M., Couvreur, F., Salvetat, J.-P., and Bouffard, S. (2002b). Micro-Raman study of the carbonization of polyimide induced by swift heavy ion irradiations. Nucl. Instrum. Methods Phys. Res. Sect. B Beam Interact. Mater. At. 194, 132-140.

Dang, C., Brandt, R.E., and Warren, S.G. (2015). Parameterizations for narrowband and broadband albedo of pure snow and snow containing mineral dust and black carbon. J. Geophys. Res. Atmospheres 120, 5446-5468.

Dartois, E., Geballe, T.R., Pino, T., Cao, A.-T., Jones, A., Deboffle, D., Guerrini, V., Bréchignac, Ph., and d'Hendecourt, L. (2007). IRAS 08572+3915: constraining the aromatic versus aliphatic content of interstellar HACs. Astron. Astrophys. 463, 635-640.

Delpoux, O., Gourier, D., Vezin, H., Binet, L., Derenne, S., and Robert, F. (2011). Biradical character of D-rich carriers in the insoluble organic matter of carbonaceous chondrites: A relic of the protoplanetary disk chemistry. Geochim. Cosmochim. Acta 75, 326-336.

Derenne, S., and Robert, F. (2010). Model of molecular structure of the insoluble organic matter isolated from Murchison meteorite: Model of molecular structure of Murchison IOM. Meteorit. Planet. Sci. 45, 1461-1475.

Doressoundiram, A., Boehnhardt, H., Tegler, S.C., and Trujillo, C. Color Properties and Trends of the Transneptunian Objects. 14.

Ehrenfreund, P., Robert, F., d'Henndecourt, L., and Béhar, F. (1991). Ehrenfreund91_AA_252_712E.pdf. 252, 712-717.

Elsila, J.E., Charnley, S.B., Burton, A.S., Glavin, D.P., and Dworkin, J.P. (2012). Compoundspecific carbon, nitrogen, and hydrogen isotopic ratios for amino acids in CM and CR chondrites and their use in evaluating potential formation pathways: Amino acid stable isotopes and formation pathways. Meteorit. Planet. Sci. 47, 1517-1536.

Ferini, G., Baratta, G.A., and Palumbo, M.E. (2004). A Raman study of ion irradiated icy mixtures. Astron. Astrophys. 414, 757-766.

Ferrari, A.C., and Robertson, J. (2000). Interpretation of Raman spectra of disordered and amorphous carbon. Phys. Rev. B 61, 14095-14107.

Ferrari, A.C., and Robertson, J. (2001). Resonant Raman spectroscopy of disordered, amorphous, and diamondlike carbon. Phys. Rev. B 64, 075414.

Foschino, S., Berné, O., and Joblin, C. (2019). Learning mid-IR emission spectra of polycyclic 
aromatic hydrocarbon populations from observations. Astron. Astrophys. 632, A84.

Gardinier, A., Derenne, S., Robert, F., Behar, F., Largeau, C., and Maquet, J. (2000). Solid state $\mathrm{CP} / \mathrm{MAS} 13 \mathrm{C}$ NMR of the insoluble organic matter of the Orgueil and Murchison meteorites: quantitative study. Earth Planet. Sci. Lett. 184, 9-21.

Ghelfi, A., Barao, F., Derome, L., and Maurin, D. (2016). Non-parametric determination of H and He interstellar fluxes from cosmic-ray data. Astron. Astrophys. 591, A94.

Godard, M., Féraud, G., Chabot, M., Carpentier, Y., Pino, T., Brunetto, R., Duprat, J., Engrand, C., Bréchignac, P., d'Hendecourt, L., et al. (2011). Ion irradiation of carbonaceous interstellar analogues: Effects of cosmic rays on the $3.4 \mu \mathrm{m}$ interstellar absorption band. Astron. Astrophys. 529, A146.

Gourier, D., Robert, F., Delpoux, O., Binet, L., Vezin, H., Moissette, A., and Derenne, S. (2008). Extreme deuterium enrichment of organic radicals in the Orgueil meteorite: Revisiting the interstellar interpretation? Geochim. Cosmochim. Acta 72, 1914-1923.

Grundy, W.M., Cruikshank, D.P., Gladstone, G.R., Howett, C.J.A., Lauer, T.R., Spencer, J.R., Summers, M.E., Buie, M.W., Earle, A.M., Ennico, K., et al. (2016). The formation of Charon's red poles from seasonally cold-trapped volatiles. Nature 539, 65-68.

Grundy, W.M., Bertrand, T., Binzel, R.P., Buie, M.W., Buratti, B.J., Cheng, A.F., Cook, J.C., Cruikshank, D.P., Devins, S.L., Dalle Ore, C.M., et al. (2018). Pluto's haze as a surface material. Icarus 314, 232-245.

Hendrix, A.R., Vilas, F., and Li, J.-Y. (2016). The UV signature of carbon in the solar system. Meteorit. Planet. Sci. 51, 105-115.

Kerridge, J.F. (1999). Formation and Processing of Organics in the Early Solar System. In Composition and Origin of Cometary Materials, K. Altwegg, P. Ehrenfreund, J. Geiss, and W.F. Huebner, eds. (Dordrecht: Springer Netherlands), pp. 275-288.

Kuga, M., Marty, B., Marrocchi, Y., and Tissandier, L. (2015). Synthesis of refractory organic matter in the ionized gas phase of the solar nebula. Proc. Natl. Acad. Sci. 112, 7129-7134.

Lacerda, P., Fornasier, S., Lellouch, E., Kiss, C., Vilenius, E., Santos-Sanz, P., Rengel, M., Müller, T., Stansberry, J., Duffard, R., et al. (2014). THE ALBEDO-COLOR DIVERSITY OF TRANSNEPTUNIAN OBJECTS. Astrophys. J. 793, L2.

Le Guillou, C., Bernard, S., Brearley, A.J., and Remusat, L. (2014). Evolution of organic matter in Orgueil, Murchison and Renazzo during parent body aqueous alteration: In situ investigations. Geochim. Cosmochim. Acta 131, 368-392.

Mathis, J.S., Rumpl, W., and Nordsieck, K.H. (1977). The size distribution of interstellar grains. Astrophys. J. 217, 425.

McKenna, A.J., Trevethan, T., Latham, C.D., Young, P.J., and Heggie, M.I. (2016). Threshold displacement energy and damage function in graphite from molecular dynamics. Carbon 99, 71-78.

Mennella, V., Palumbo, M.E., and Baratta, G.A. (2004). Formation of CO and CO2 molecules by ion irradiation of water ice-covered hydrogenated carbon grains. Astrophys. J. 615, 1073.

Merlin, F., Guilbert, A., Dumas, C., Barucci, M.A., de Bergh, C., and Vernazza, P. (2007). Properties of the icy surface of the TNO 136108 (2003 EL\$_\{\sf 61\}\$). Astron. Astrophys. 466, 1185-1188.

Merlin, F., Hromakina, T., Perna, D., Hong, M.J., and Alvarez-Candal, A. (2017). Taxonomy of trans-Neptunian objects and Centaurs as seen from spectroscopy. Astron. Astrophys. 604, A86.

Mewaldt, R.A., Cohen, C.M.S., Mason, G.M., Haggerty, D.K., and Desai, M.I. (2007a). Long-Term Fluences of Solar Energetic Particles from H to Fe. Space Sci. Rev. 130, 323-328.

Mewaldt, R.A., Cohen, C.M.S., Mason, G.M., Cummings, A.C., Desai, M.I., Leske, R.A., Raines, J., Stone, E.C., Wiedenbeck, M.E., von Rosenvinge, T.T., et al. (2007b). On the Differences in Composition between Solar Energetic Particles and Solar Wind. Space Sci. Rev. 130, 207-219.

Meyer, J.-P., Drury, L.O., and Ellison, D.C. (1998). A Cosmic-Ray Composition Controlled by Volatility and A/Q Ratio. SNR Shock Acceleration of Gas and Dust. In The Advanced Composition Explorer Mission, C.T. Russell, R.A. Mewaldt, and T.T. Von Rosenvinge, eds. (Dordrecht: Springer Netherlands), pp. 179-201. 
Miyazawa, T., Fukushima, K., and Ideguchi, Y. (1962). Molecular Vibrations and Structure of High Polymers. ITI. Polarized Infrared Spectra, Normal Vibrations, and Helical Conformation of Polyethylene Glycol. 37, 2764-2776.

Nakai, K., Kinoshita, C., and Matsunaga, A. (1991). A study of amorphization and microstructural evolution of graphite under electron or ion irradiation. Ultramicroscopy 39, 361-368.

Olejniczak, A., and Skuratov, V.A. (2014). Effect of swift heavy ion irradiation on single- and multiwalled carbon nanotubes. Nucl. Instrum. Methods Phys. Res. Sect. B Beam Interact. Mater. At. 326, 33-36.

Parise, B., Ceccarelli, C., Tielens, A.G.G.M., Castets, A., Caux, E., Lefloch, B., and Maret, S. (2006). Testing grain surface chemistry: a survey of deuterated formaldehyde and methanol in lowmass class 0 protostars. Astron. Astrophys. 453, 949-958.

Portugal, W., Pilling, S., Boduch, P., Rothard, H., and Andrade, D.P.P. (2014). Radiolysis of amino acids by heavy and energetic cosmic ray analogues in simulated space environments: $\alpha$-glycine zwitterion form. Mon. Not. R. Astron. Soc. 441, 3209-3225.

Quirico, E., Montagnac, G., Rouzaud, J.-N., Bonal, L., Bourot-Denise, M., Duber, S., and Reynard, B. (2009). Precursor and metamorphic condition effects on Raman spectra of poorly ordered carbonaceous matter in chondrites and coals. Earth Planet. Sci. Lett. 287, 185-193.

Quirico, E., Orthous-Daunay, F.-R., Beck, P., Bonal, L., Brunetto, R., Dartois, E., Pino, T., Montagnac, G., Rouzaud, J.-N., Engrand, C., et al. (2014). Origin of insoluble organic matter in type 1 and 2 chondrites: New clues, new questions. Geochim. Cosmochim. Acta 136, 80-99.

Quirico, E., Bonal, L., Montagnac, G., Beck, P., and Reynard, B. (2020). New insights into the structure and formation of coals, terrestrial and extraterrestrial kerogens from resonant UV Raman spectroscopy. Geochim. Cosmochim. Acta 282, 156-176.

Remusat, L., Derenne, S., and Robert, F. (2005a). New insight on aliphatic linkages in the macromolecular organic fraction of Orgueil and Murchison meteorites through ruthenium tetroxide oxidation. Geochim. Cosmochim. Acta 69, 4377-4386.

Remusat, L., Derenne, S., Robert, F., and Knicker, H. (2005b). New pyrolytic and spectroscopic data on Orgueil and Murchison insoluble organic matter: A different origin than soluble? Geochim. Cosmochim. Acta 69, 3919-3932.

Remusat, L., Palhol, F., Robert, F., Derenne, S., and Francelanord, C. (2006). Enrichment of deuterium in insoluble organic matter from primitive meteorites: A solar system origin? Earth Planet. Sci. Lett. 243, 15-25.

Ribas, I., Guinan, E.F., Güdel, M., and Audard, M. (2005). Evolution of the solar activity over time and effects onn planetary atmospheres. I. High-energy irradiances (1-1700 A). 622, 680694.

Robert, F., and Epstein, S. (1982). The concentration and isotopic composition of hydrogen, carbon and nitrogen in carbonaceous meteorites. Geochim. Cosmochim. Acta 46, 81-95.

Roberts, H., and Millar, T.J. (2000). Gas-phase formation of doubly-deuterated species. 364, 780-784.

Robertson, J. (1995). Structural models of a-C and a-C:H. Diam. Relat. Mater. 4, 297-301.

Schmidt, J.S., and Hinrichs, R. (2020). Evaluation of terrestrial carbonaceous matter aromatization by Raman spectroscopy and its application to $\mathrm{C}$ chondrites. Meteorit. Planet. Sci. 55, 800-817.

Schmitt, B., Philippe, S., Grundy, W.M., Reuter, D.C., Côte, R., Quirico, E., Protopapa, S., Young, L.A., Binzel, R.P., Cook, J.C., et al. (2017). Physical state and distribution of materials at the surface of Pluto from New Horizons LEISA imaging spectrometer. Icarus 287, 229-260.

Schmitt-Kopplin, P., Gabelica, Z., Gougeon, R.D., Fekete, A., Kanawati, B., Harir, M., Gebefuegi, I., Eckel, G., and Hertkorn, N. (2010). High molecular diversity of extraterrestrial organic matter in Murchison meteorite revealed 40 years after its fall. Proc. Natl. Acad. Sci. 107, 2763-2768.

Sekine, Y., Genda, H., Kamata, S., and Funatsu, T. (2017). The Charon-forming giant impact as a source of Pluto's dark equatorial regions. Nat. Astron. 1, 0031.

Seperuelo Duarte, E., Domaracka, A., Boduch, P., Rothard, H., Dartois, E., and da Silveira, E.F. (2010). Laboratory simulation of heavy-ion cosmic-ray interaction with condensed CO. 
Astron. Astrophys. 512, A71.

Sephton, M.A. (2002). Organic compounds in carbonaceous meteorites. Nat. Prod. Rep. 19, 292-311.

Severin, D., Ensinger, W., Neumann, R., Trautmann, C., Walter, G., Alig, I., and Dudkin, S. (2005). Degradation of polyimide under irradiation with swift heavy ions. Nucl. Instrum. Methods Phys. Res. Sect. B Beam Interact. Mater. At. 236, 456-460.

Severin, D., Balanzat, E., Ensinger, W., and Trautmann, C. (2010). Outgassing and degradation of polyimide induced by swift heavy ion irradiation at cryogenic temperature. J. Appl. Phys. $108,024901$.

Stanishevsky, A. (2001). Patterning of diamond and amorphous carbon films using focused ion beams. Thin Solid Films 398-399, 560-565.

Stern, S.A., Bagenal, F., Ennico, K., Gladstone, G.R., Grundy, W.M., McKinnon, W.B., Moore, J.M., Olkin, C.B., Spencer, J.R., Weaver, H.A., et al. (2015). The Pluto system: Initial results from its exploration by New Horizons. Science 350, aad1815-aad1815.

Ullmann, J., Delan, A., and Schmidt, G. (1993). Ion etching behaviour and surface binding energies of hard diamond-like carbon and microwave chemical vapour deposition diamond films. Diam. Relat. Mater. 2, 266-271.

Urso, R.G., Baklouti, D., Djouadi, Z., Pinilla-Alonso, N., and Brunetto, R. (2020). Near-infrared Methanol Bands Probe Energetic Processing of Icy Outer Solar System Objects. Astrophys. J. 894, L3.

Usoskin, I.G., Alanko-Huotari, K., Kovaltsov, G.A., and Mursula, K. (2005). Heliospheric modulation of cosmic rays: Monthly reconstruction for 1951-2004. J. Geophys. Res. 110, A12108.

Visser, R., Bruderer, S., Cazzoletti, P., Facchini, S., Heays, A.N., and van Dishoeck, E.F. (2018). Nitrogen isotope fractionation in protoplanetary disks. Astron. Astrophys. 615, A75.

Vuković, F., Leyssale, J.-M., Aurel, P., and Marks, N.A. (2018). Evolution of Threshold Displacement Energy in Irradiated Graphite. Phys. Rev. Appl. 10, 064040.

Wang, Y., Huang, Y., Alexander, C.M.O. 'D., Fogel, M., and Cody, G. (2005). Molecular and compound-specific hydrogen isotope analyses of insoluble organic matter from different carbonaceous chondrite groups. Geochim. Cosmochim. Acta 69, 3711-3721.

Watanabe, N., and Kouchi, A. (2008). Ice surface reactions: A key to chemical evolution in space. Prog. Surf. Sci. 83, 439-489.

Webber, W.R., and Yushak, S.M. (1983). A measurement of the energy spectra and relative abundance of the cosmic-ray $\mathrm{H}$ and He isotopes over a broad energy range. Astrophys. J. 275, 391.

Wooden, D. (2000). Mg-Rich Silicate Crystals in Comet Hale-Bopp: ISM Relics or Solar Nebula Condensates? Icarus 143, 126-137.

Yun, Y., Ma, X., Gui, J., Broitman, E., and Gellman, A.J. (2007). Oxidation Kinetics of Hydrogenated Amorphous Carbon ( $a-\mathrm{CH} \times$ ) Overcoats for Magnetic Data Storage Media. Langmuir 23, 5485-5490.

Zhai, P.-F., Liu, J., Zeng, J., Yao, H.-J., Duan, J.-L., Hou, M.-D., Sun, Y.-M., and Charles, E.R. (2014). Raman spectrum study of graphite irradiated by swift heavy ions. Chin. Phys. B 23, 126105.

Zolensky, M.E., Zega, T.J., Yano, H., Wirick, S., Westphal, A.J., Weisberg, M.K., Weber, I., Warren, J.L., Velbel, M.A., Tsuchiyama, A., et al. (2006). Mineralogy and Petrology of Comet 81P/Wild 2 Nucleus Samples. Science 314, 1735-1739.

Zubko, V.G., Mennella, V., Colangeli, L., and Bussoletti, E. (1996). Optical constants of cosmic carbon analogue grains - I. Simulation of clustering by a modified continuous distribution of ellipsoids. Mon. Not. R. Astron. Soc. 282, 1321-1329. 

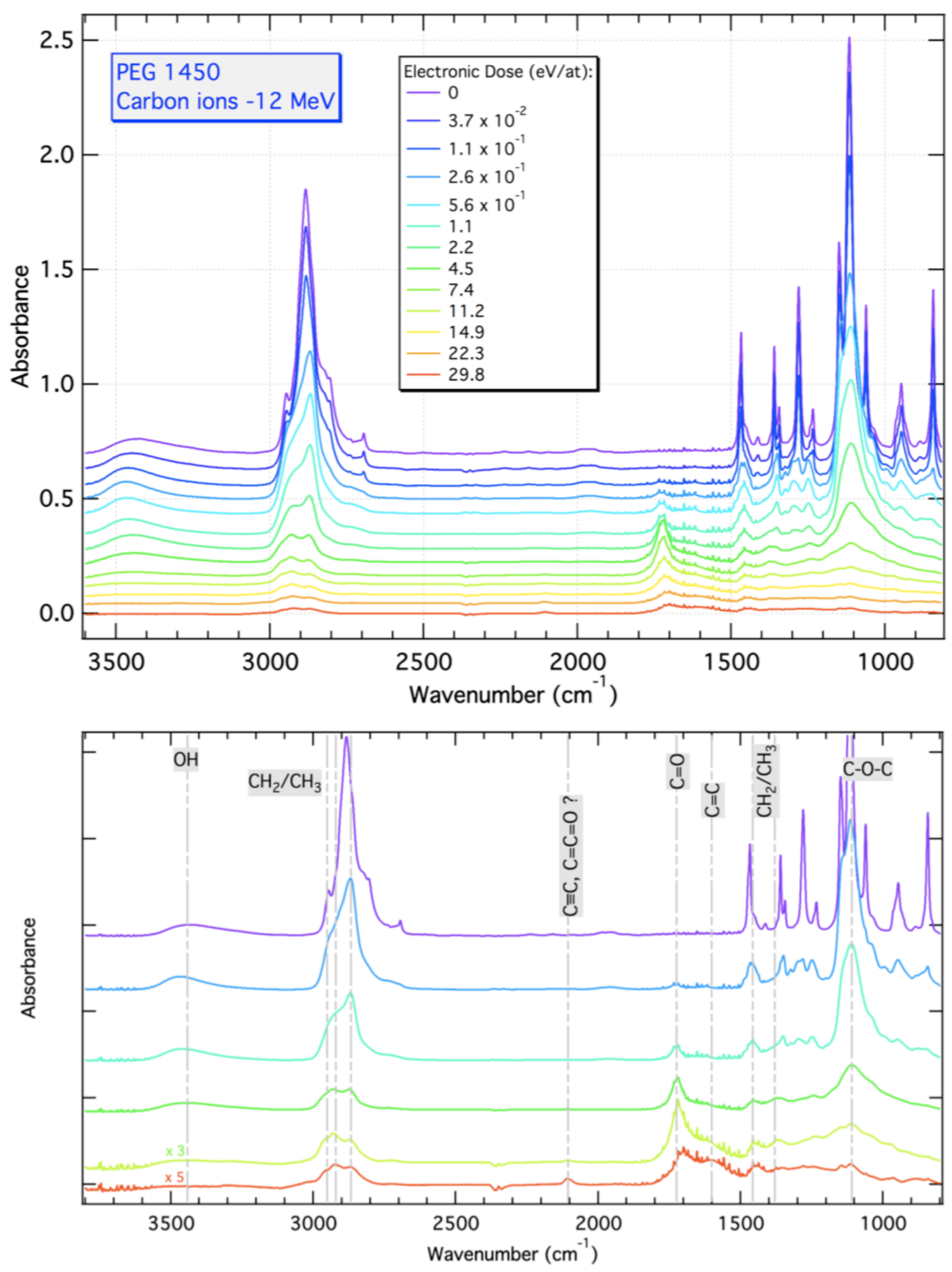

Figure 1: Infrared spectra of irradiated PEG1450 with $12 \mathrm{MeV}$ Carbon ions. Spectra are offshifted for the sake of clarity. 


\section{FIGURE 2}

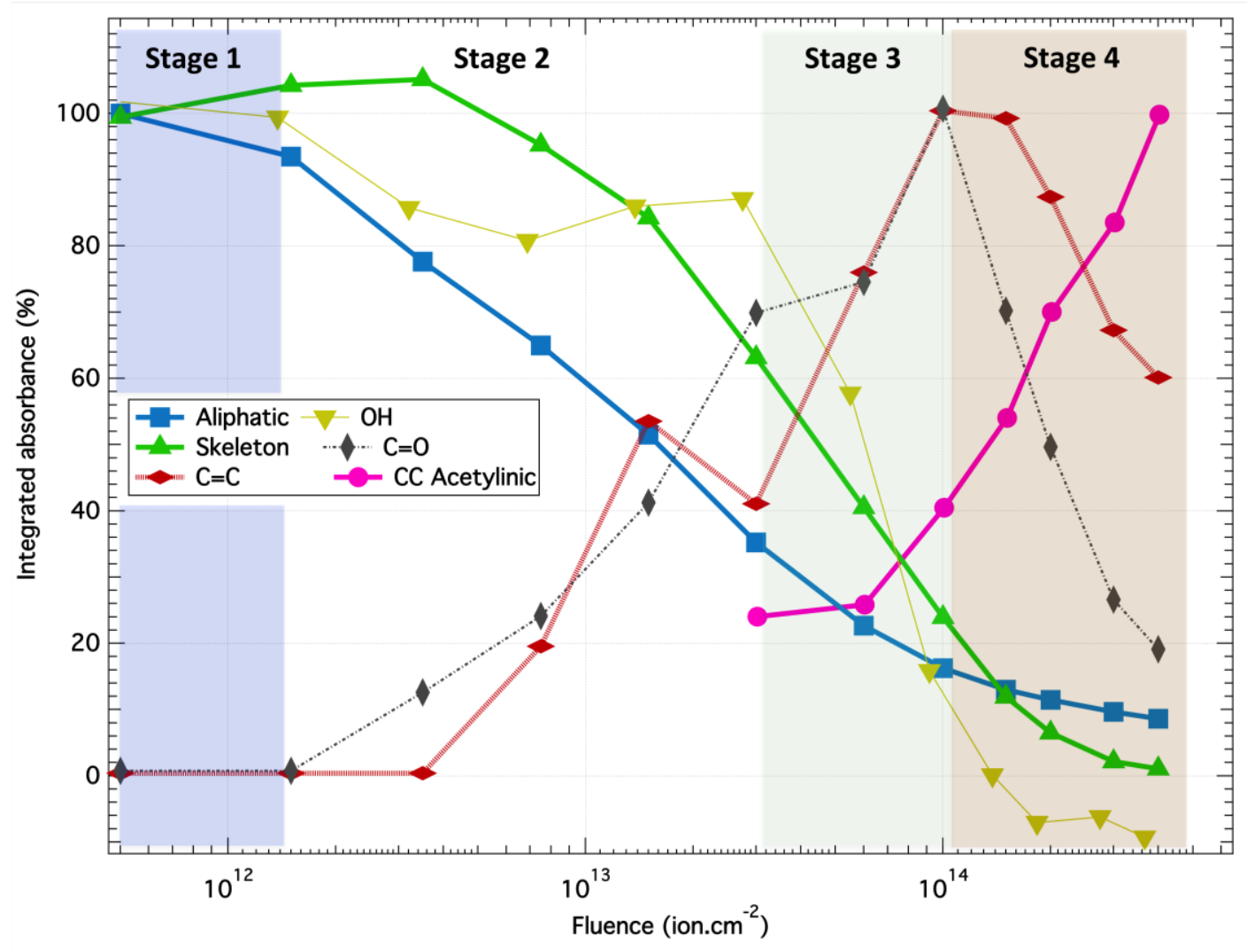

Figure 2: Normalized integrated absorbance of the main chemical groups in pristine and irradiated PEG1450. Four stages are identified from these curves (see text). 


\section{FIGURE 3}
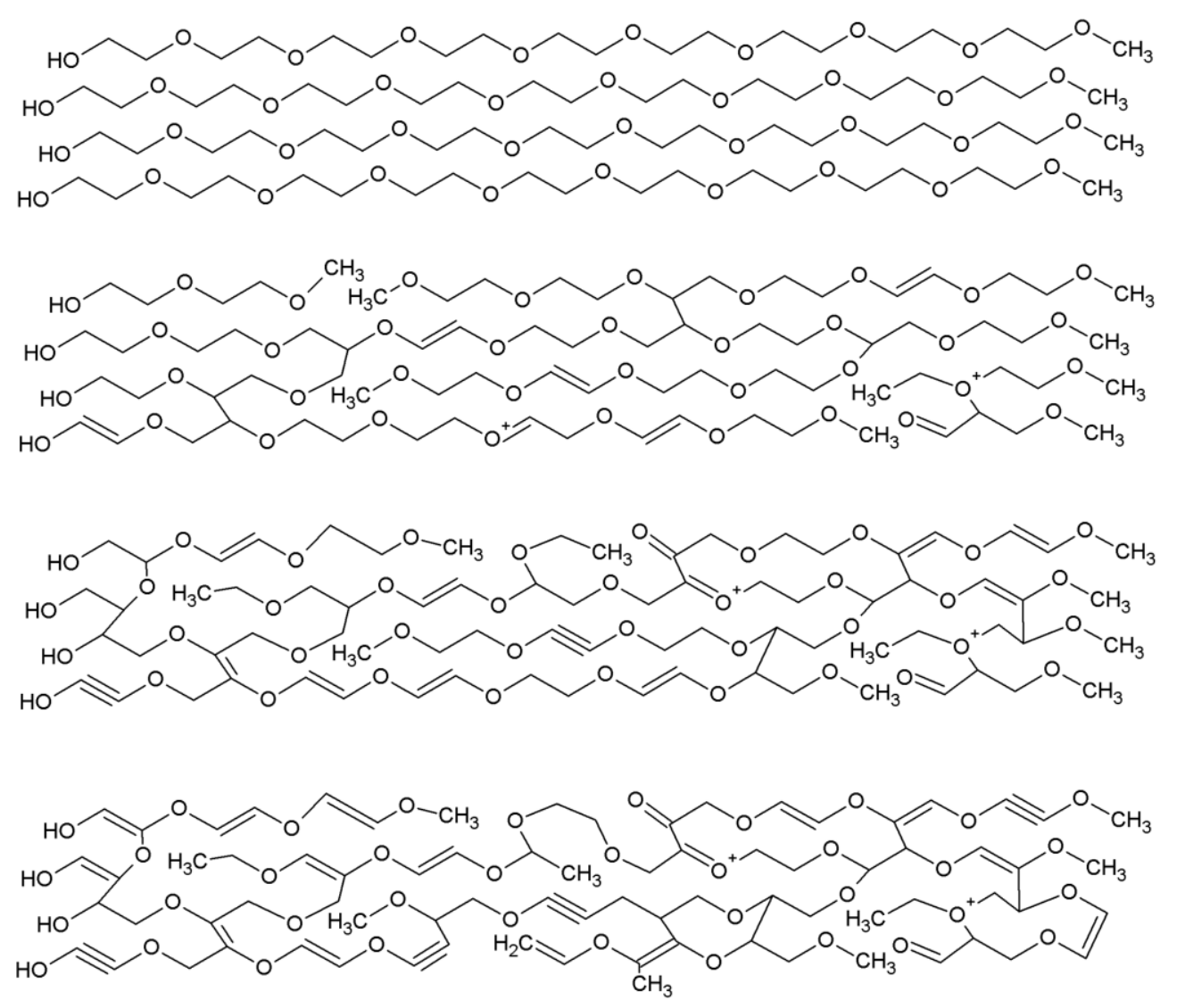

Stage 4

Figure 3: Schematic view of the chemical and structural evolution of PEG1450 under irradiation with $12 \mathrm{MeV}$ carbon ions. The arrow points to the increasing dose up to 32 eV.atom-1 $\left(410^{14} \mathrm{~cm}^{-2}\right)$. 


\section{FIGURE 4}

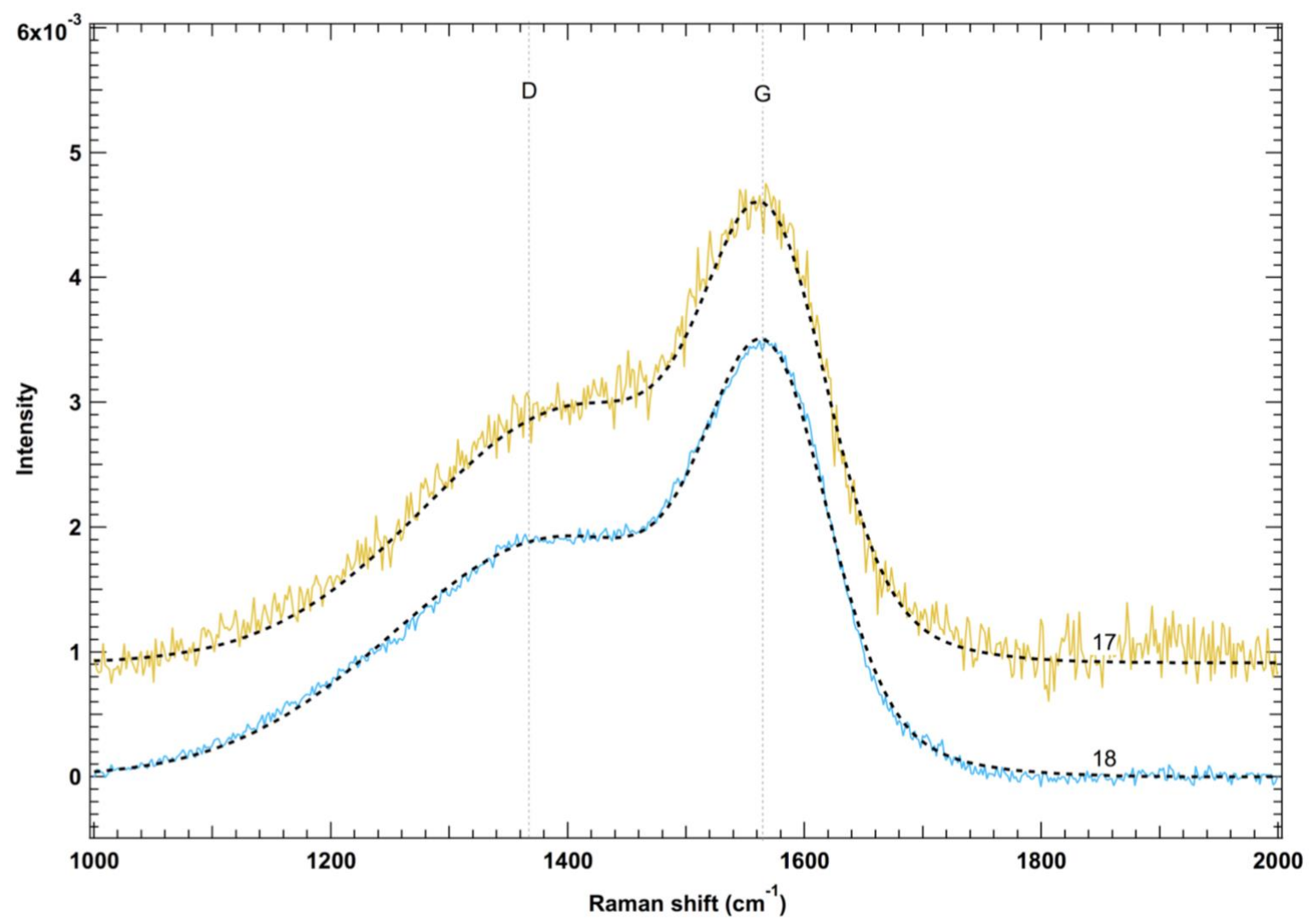

Figure 4: Visible Raman spectra $(\lambda=532 \mathrm{~nm})$, after fluorescent background subtraction, of cellulose (17) and lignin (18) irradiated with $10 \mathrm{MeV}$ Nickel ions at fluences of $5.40 .10^{13}$ and $7.10^{13}$ ions.cm ${ }^{-2}$, respectively (see Table 1 for detailed experimental conditions). The dash lines represent the two Gaussian fits. Spectra are shifted for clarity and the $\mathrm{G}$ and $\mathrm{D}$ bands $\mathrm{D}$ are marked with vertical lines. 


\section{FIGURE 5}

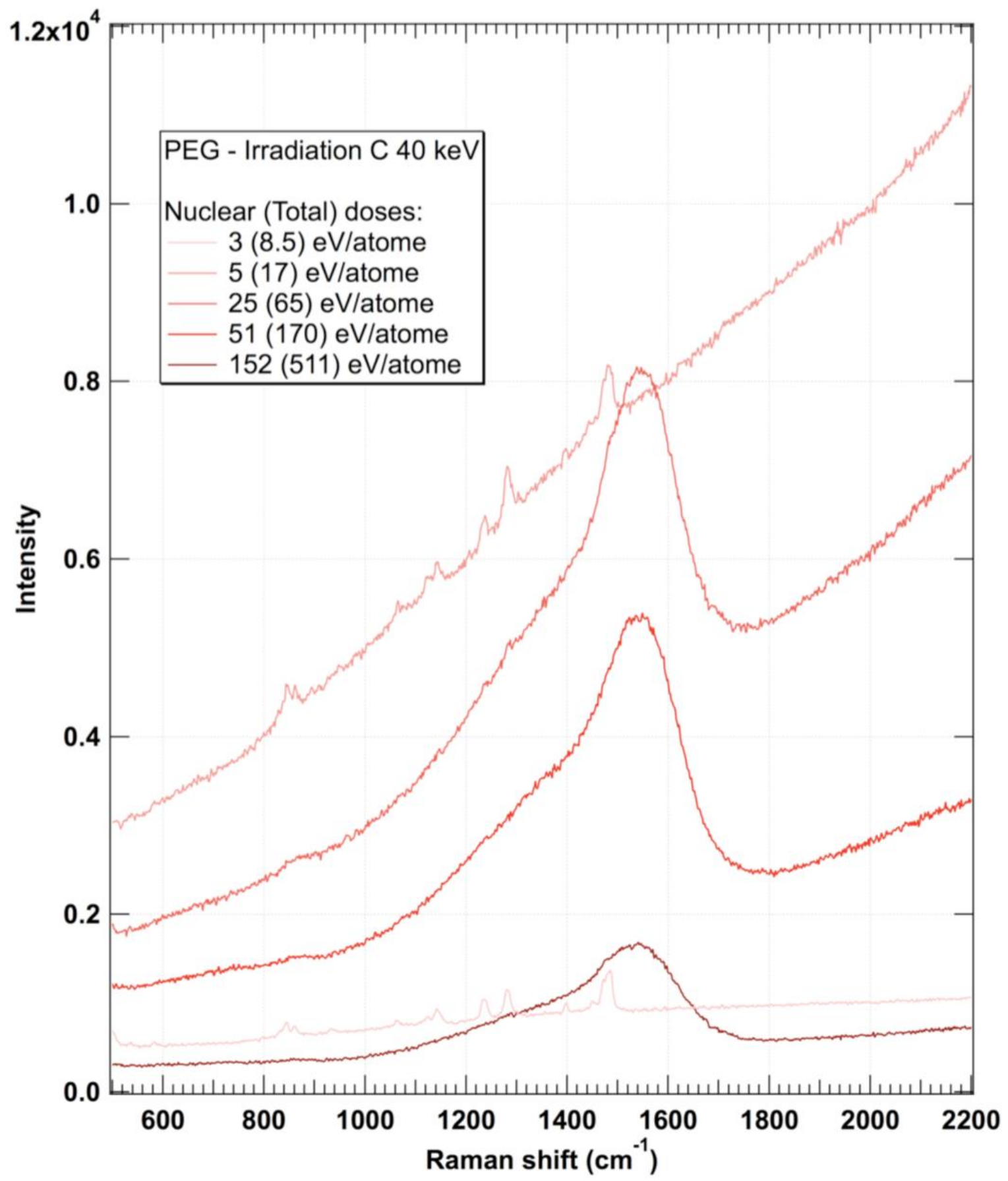

Figure 5: Visible Raman spectra $(\lambda=532 \mathrm{~nm})$ of PEG 1450 irradiated $40 \mathrm{keV}$ C ions. Note the progressive appearance of the first-order carbon bands. 


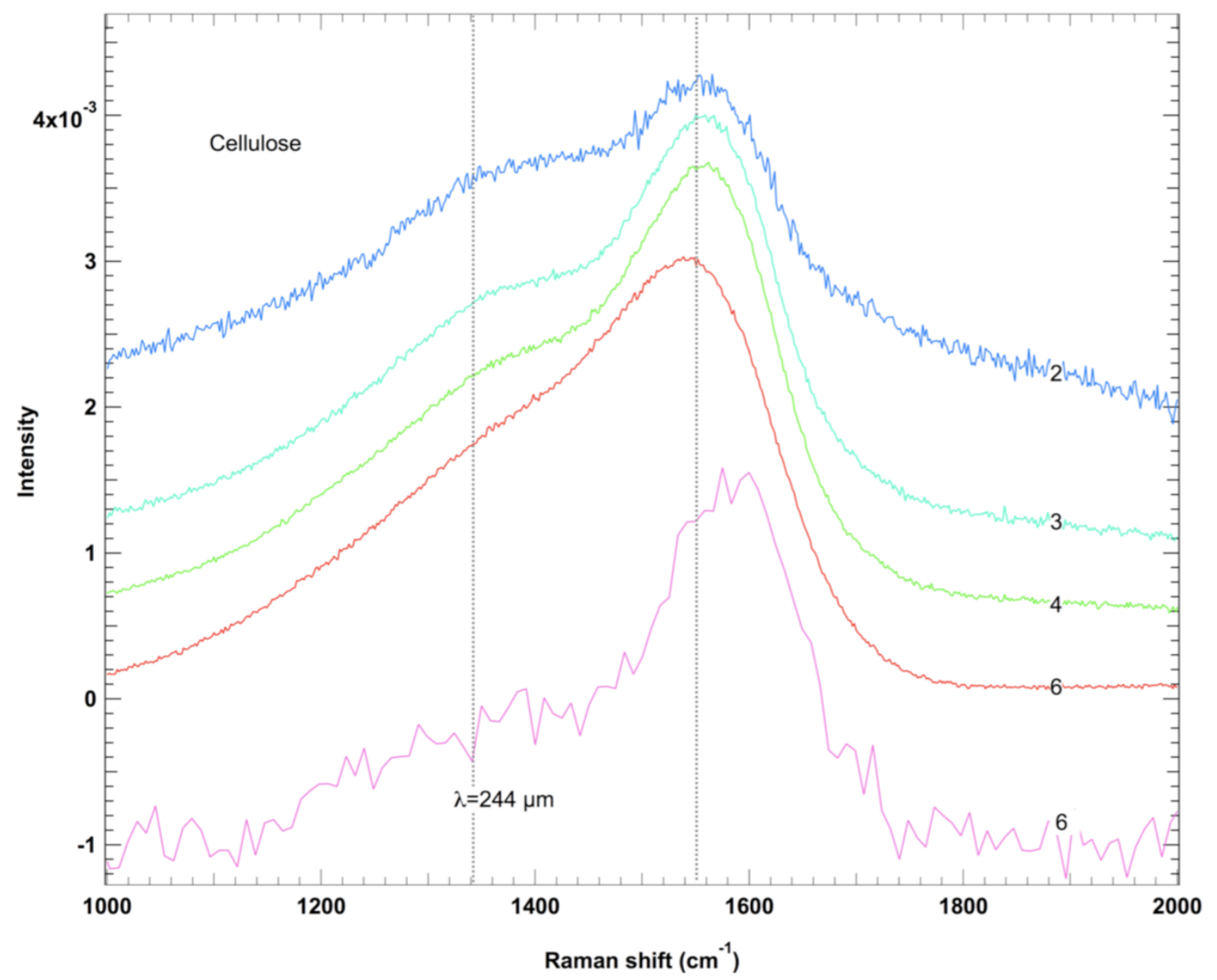

Figure 6: Visible Raman spectra $(\lambda=532 \mathrm{~nm})$ in the range $\left[1000 \mathrm{~cm}^{-1}, 2000 \mathrm{~cm}^{-1}\right]$ of 5 cellulose irradiated with $170 \mathrm{keV}$ Neon. For each precursor, the highest spectrum (blue) 6 is obtained at the nuclear dose from which the D and G bands appear, and then 7 downwards, those obtained for increasing doses. UV Raman spectrum $(\lambda=244 \mathrm{~nm})$ is 8 also shown (c). The numbers refer to those indicated in Table 1. Spectra are vertically 9 shifted for the sake of clarity. 

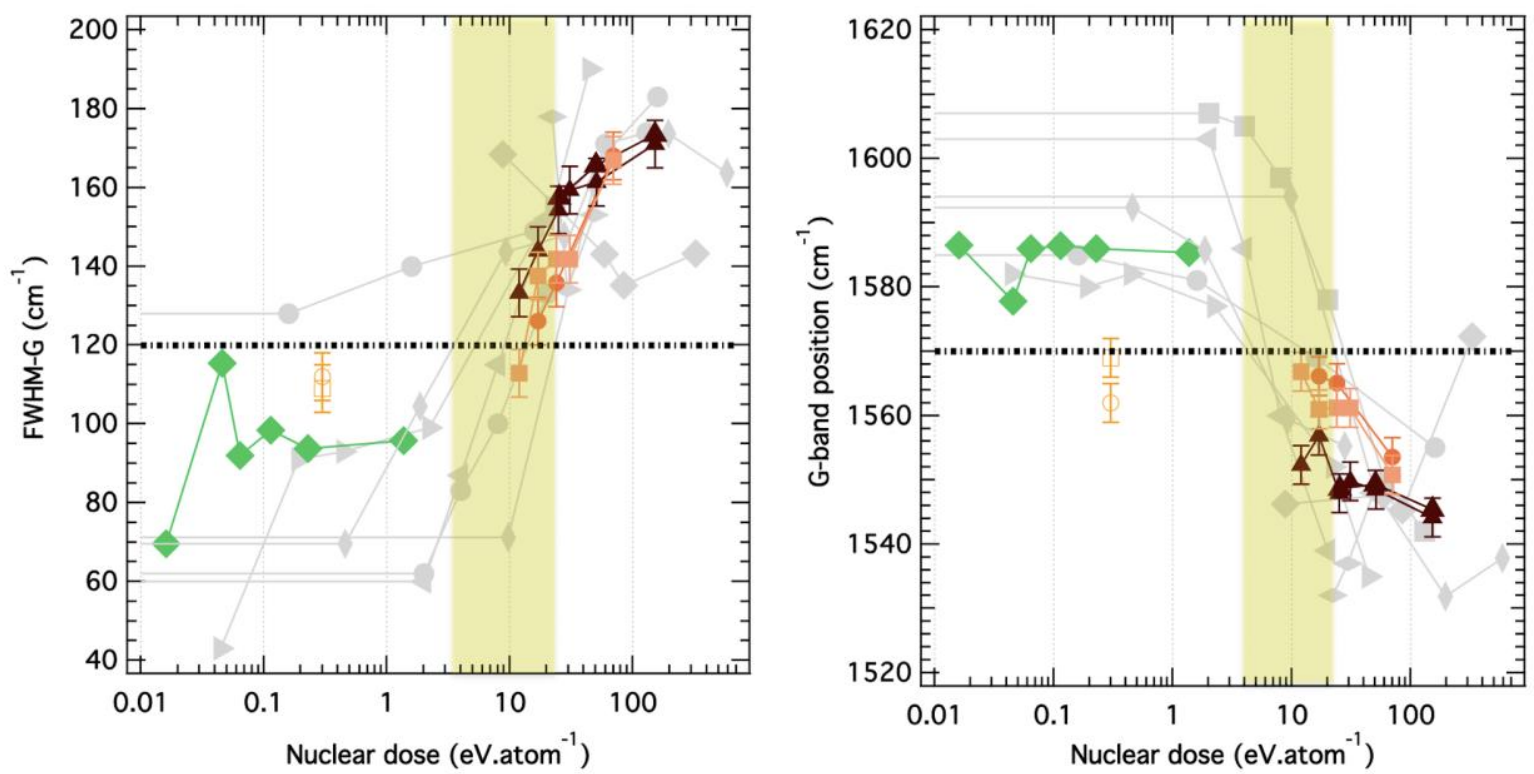



High energy implantations: Lignin Cellulose
Aliphatic soots (Brunetto et al., 2009)

Aromatic soots (Brunetto et al., 2009)

Allende (Brunetto et al., 2014)

Amorphous Carbon (Baratta et al., 2004)

Highly Oriented Pyrolyzed Graphite (Baratta et al., 2004)

Ice mixture (Ferini et al., 2004)

Benzene (Strazzula and Baratta, 1992)

Kapton H (Costantini et al., 2000)

Figure 7: G band full width at half maximum (FWHM-G) (left) and G band peak position (right) as a function of the nuclear dose for different carbonaceous precursors from this work and from the literature (Tab. 4). The G band shape is largely modified for a nuclear 


\section{FIGURE 8}
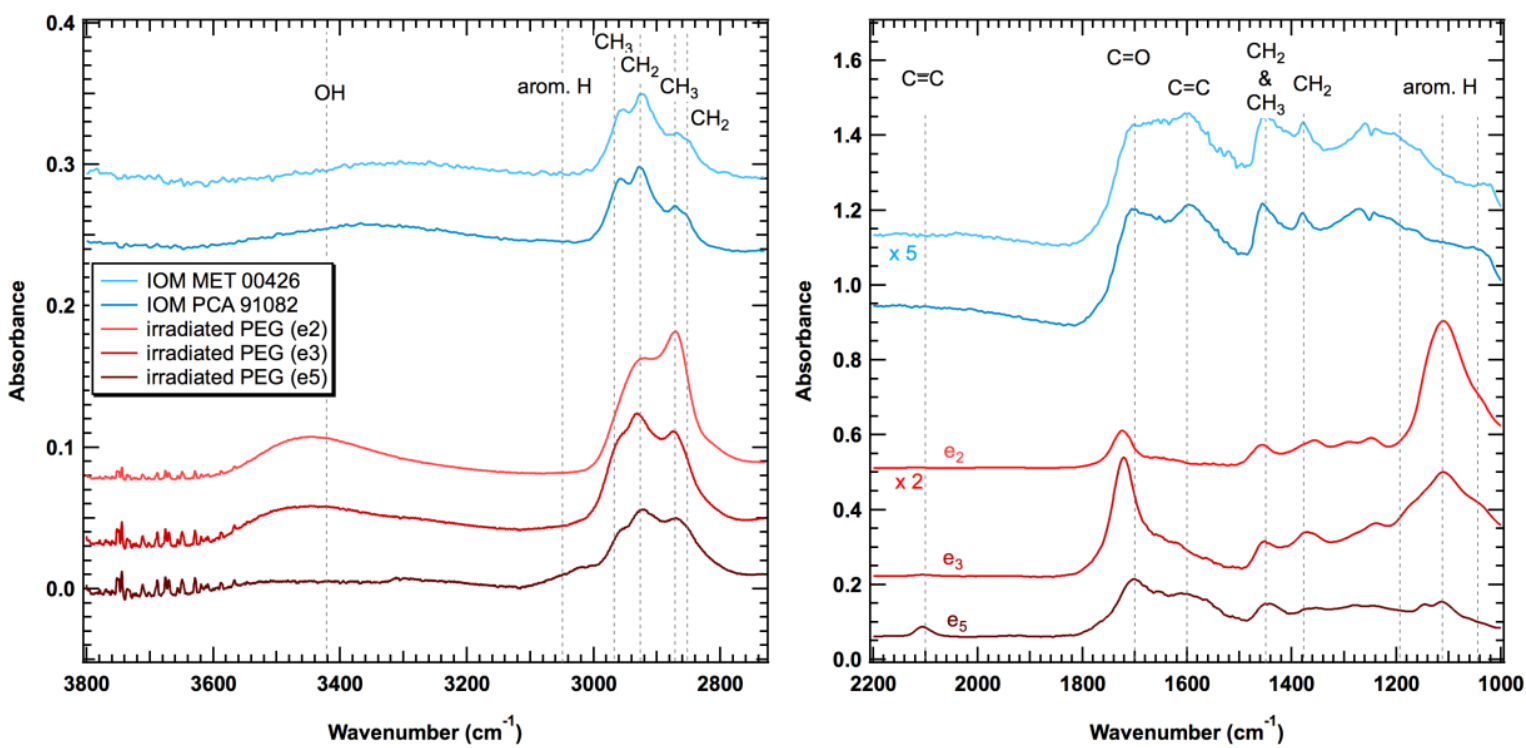

Figure 8: Infrared spectra of irradiated PEG at high energy compared with spectra of least processed meteorites by secondary parent body processes (Abreu and Brearley, 


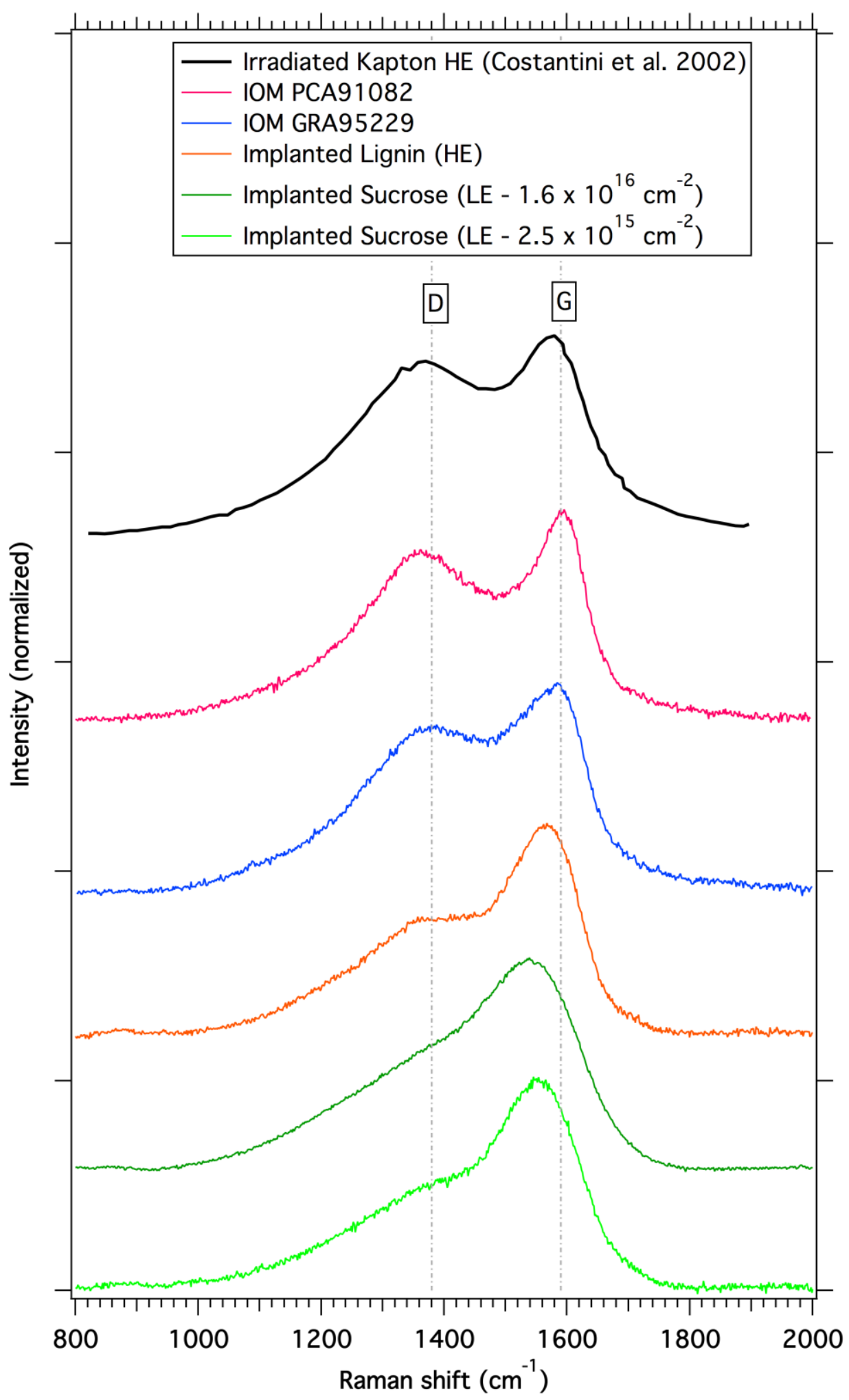

Figure 9: $532 \mathrm{~nm}$ Raman spectra spectra of implanted samples withh high and low energy ions, compared with spectra of IOM samples extracted from two primitive CR chondrites, and a Kapton $\mathrm{H}$ polymer irradiated with swift heavy ions. 
53

54

55

\section{FIGURE A1}
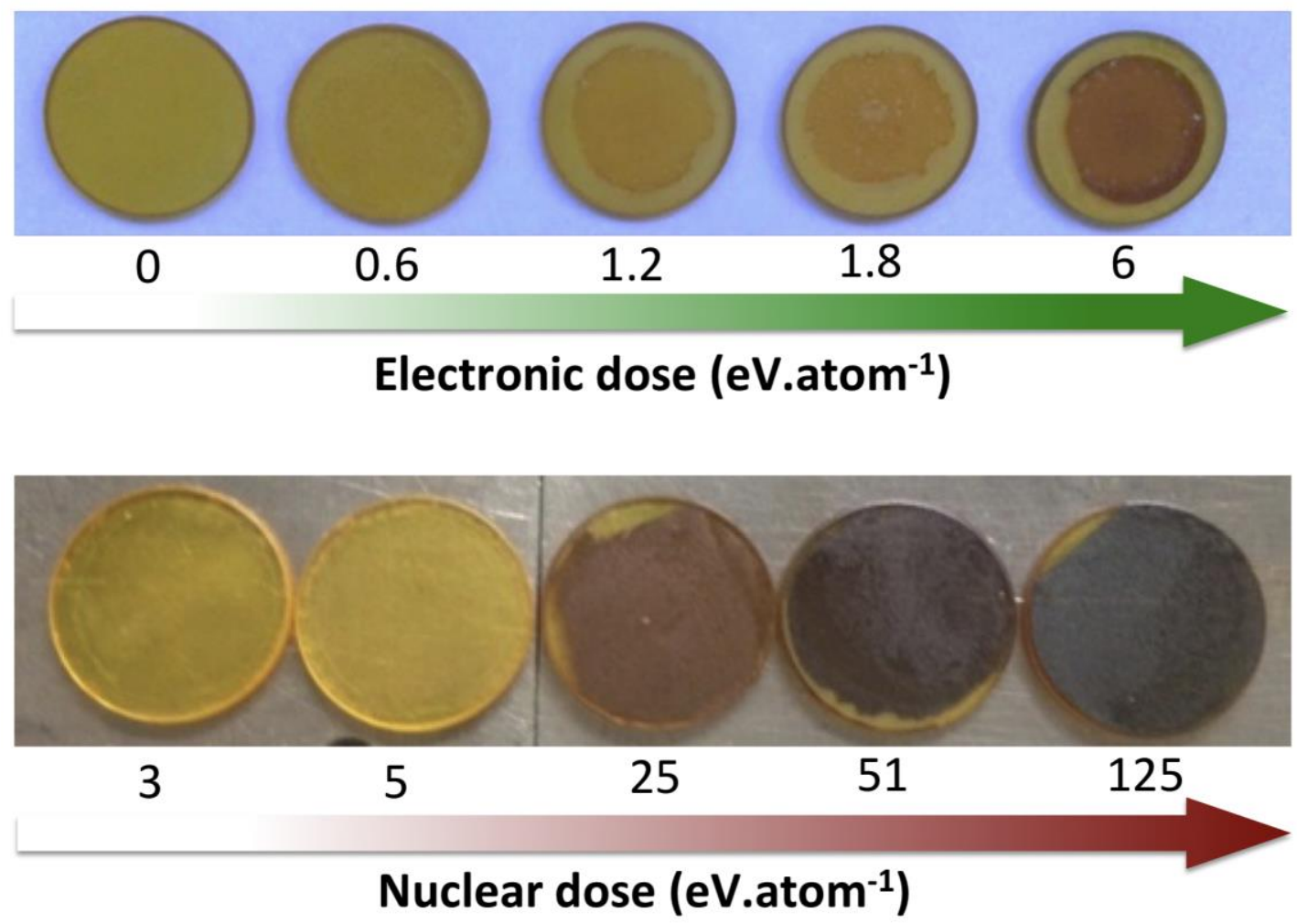

Figure A1: Top: PEG1450 irradiated with $12 \mathrm{MeV}$ Carbon ions. The starting transparent film gets darker, turning from yellow to brown-red. Bottom: PEG1450 irradiated with 40

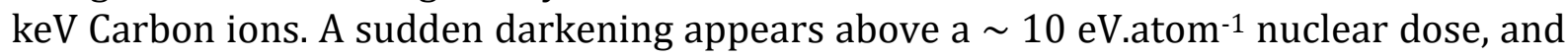
then the film turned to black. 

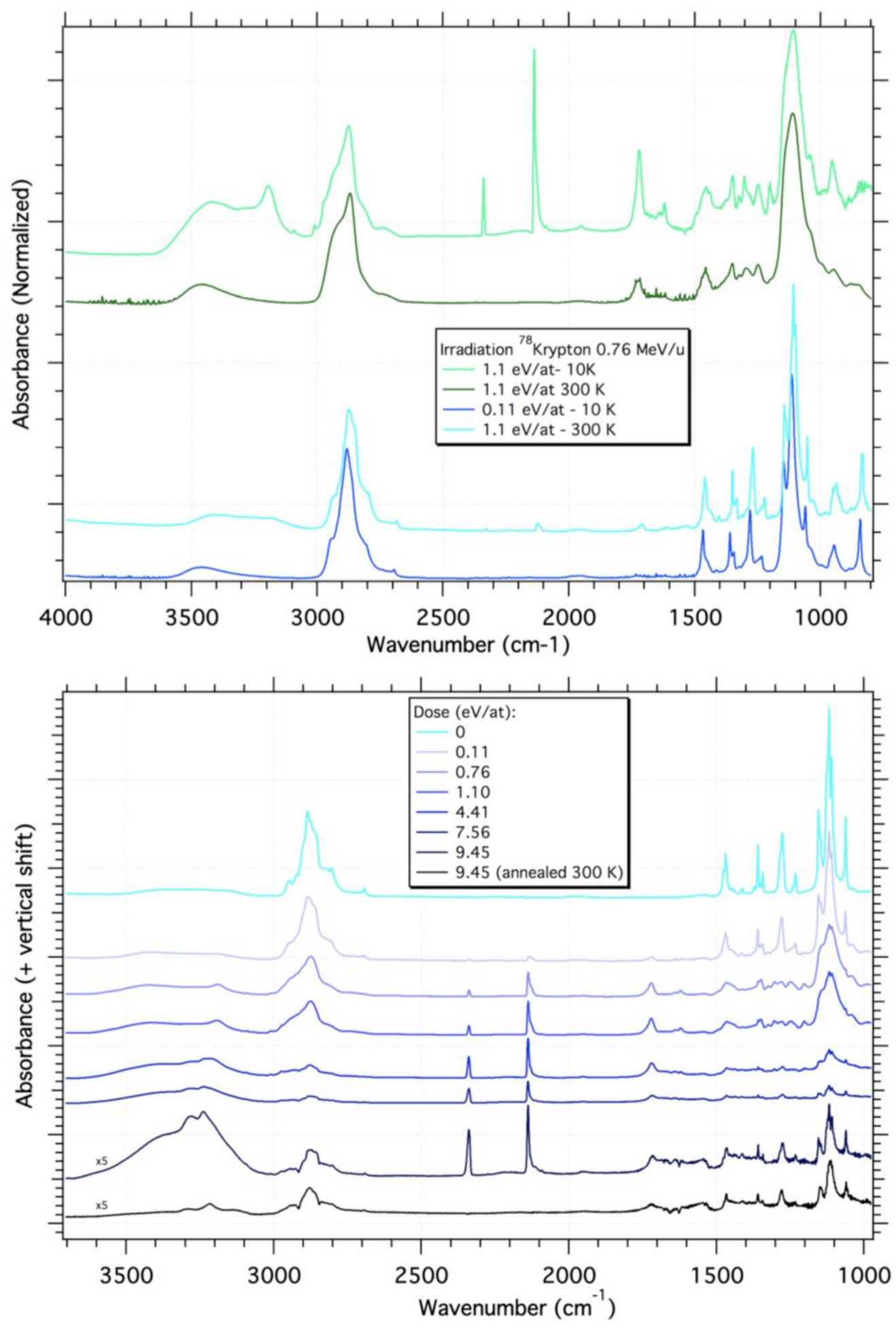

Figure A2: Infrared spectra of PEG 1450 irradiated at $10 \mathrm{~K}$ with $59 \mathrm{MeV} \mathrm{Kr}$. At 0.1 and $1.1 \mathrm{eV} /$ atom, the spectra are very similar to those of PEG irradiated at room temperature, except the presence of volatile species $\left(\mathrm{CO}, \mathrm{CO}_{2}, \mathrm{CH}_{4}, \mathrm{H}_{2} \mathrm{O}\right)$ and the region $70 \quad 3600-3000 \mathrm{~cm}^{-1}$ (see text). 


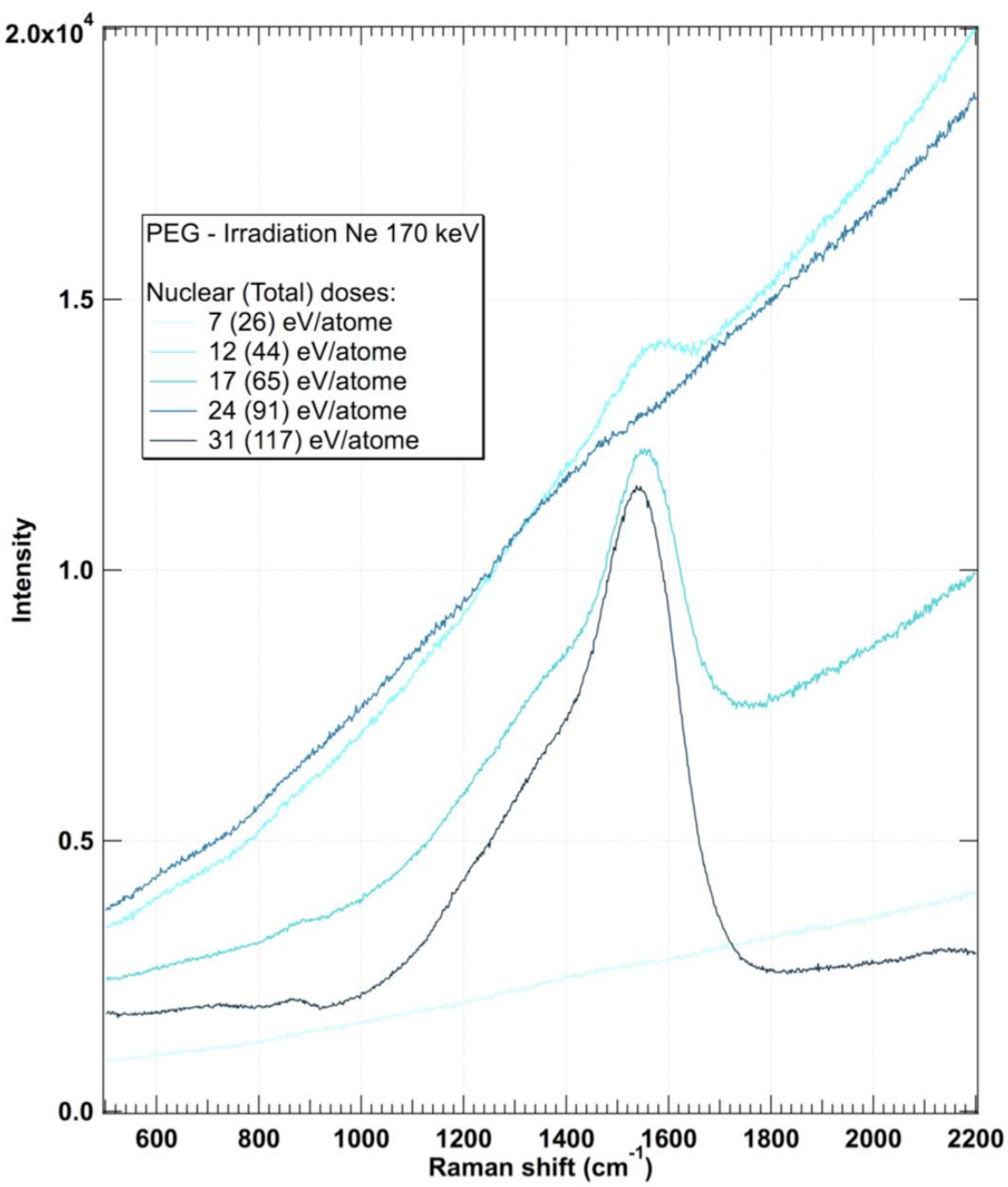

74 Figure A3: Visible Raman spectra $(\lambda=532 \mathrm{~nm})$ of PEG 1450 irradiated with $170 \mathrm{keV} \mathrm{Ne}$

75 Note the progressive appearance of the first-order carbon bands. 

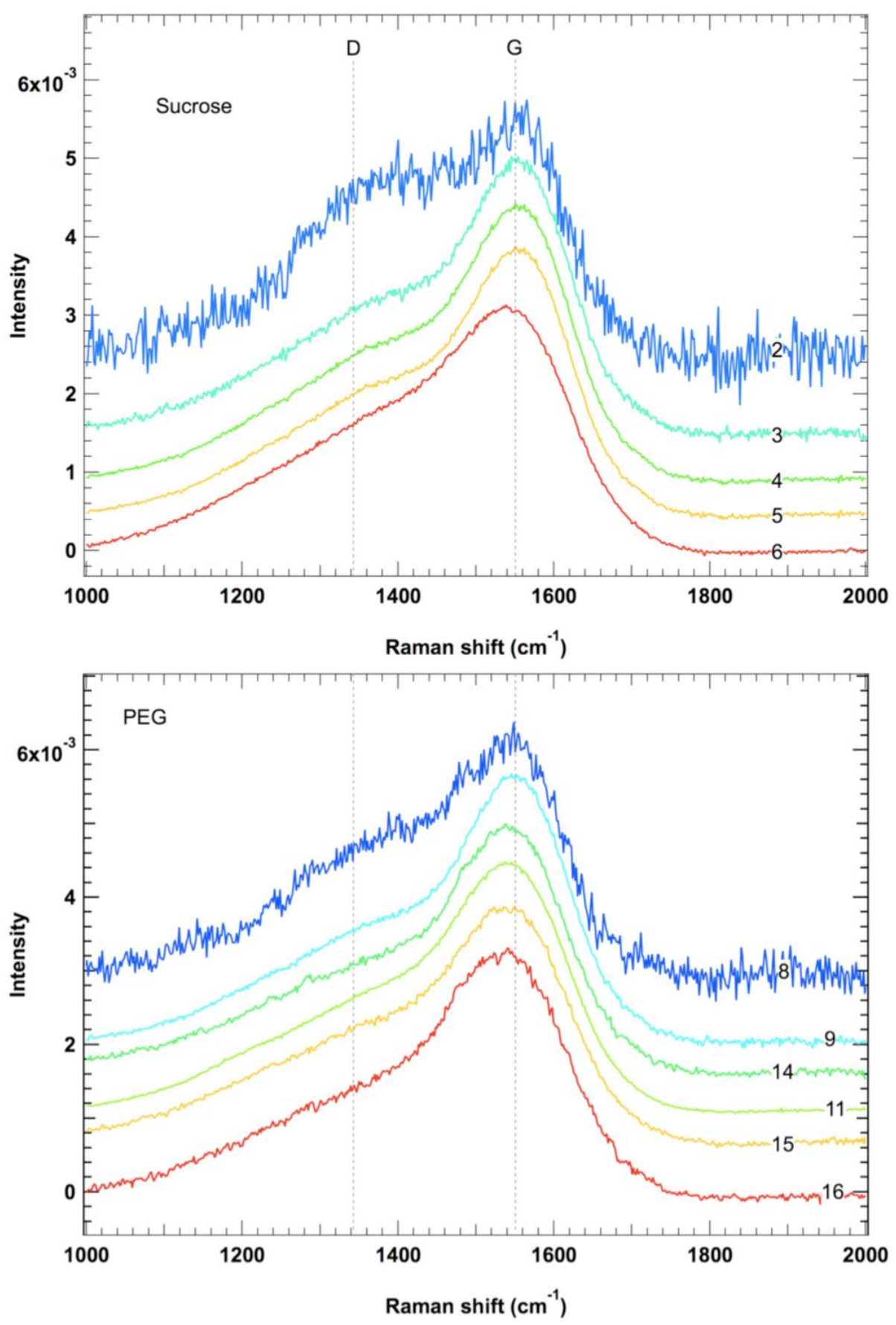

Figure A4: Visible Raman spectra $(\lambda=532 \mathrm{~nm})$ in the range $1000 \mathrm{~cm}^{-1}, 2000 \mathrm{~cm}^{-1}$ of sucrose and PEG irradiated with $170 \mathrm{keV}$ Neon and $40 \mathrm{keV}$ Carbonn. For each precursor, the highest spectrum (blue) is obtained at the nuclear dose from which the D and G bands appear, and then downwards, those obtained for increasing doses. The numbers refer to those indicated in Table 1 . Spectra are vertically shifted for the sake of clarity. 


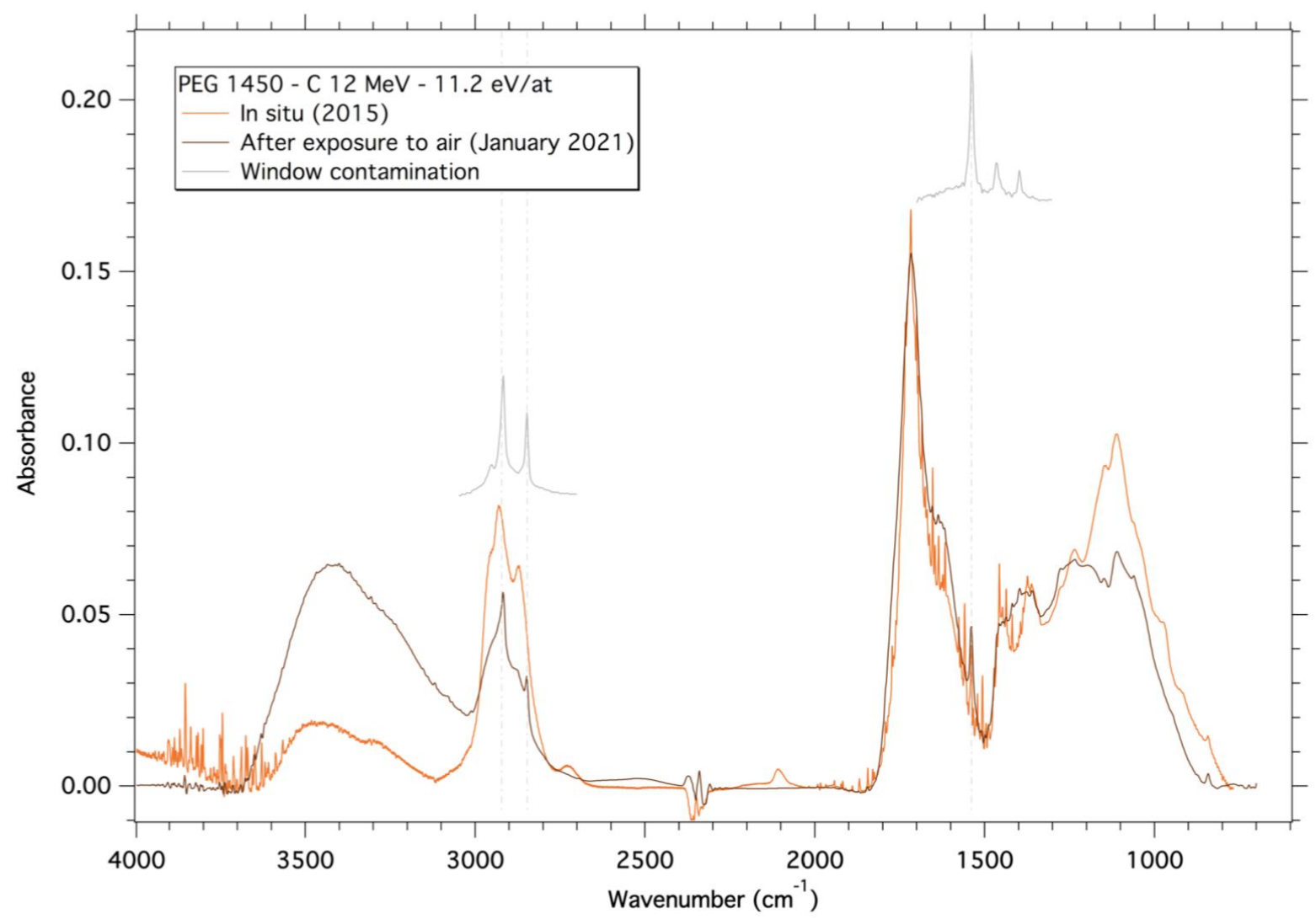

Figure A5: Infrared spectra of PEG 1450 irradiated with $12 \mathrm{MeV} \mathrm{C}$ ions collected in situ 88 under vacuum and after exposure to air and long-duration storage. Impact of air 89 exposure mostly results in a decrease of aliphatic bands and of the peak at $\sim 1100 \mathrm{~cm}^{-1}$. 90 Note that the spectrum collected under air points to an organic contamination of the 91 window that slightly blur the aliphatic band in the range $2800-3000 \mathrm{~cm}^{-1}$. 